\title{
A Systematic Study of the Best Fitting Line Problem
}

\author{
Giorgio Gambirasio,giga@uol.com.br
}

Denise Consonni, denise.consonni@gmail.com

\begin{abstract}
The classical approach for tackling the problem of drawing the 'best fitting line' through a plot of experimental points (here called a scenario) is the least square process applied to the errors along the vertical axis. However, more elaborate processes exist or may be found. In this report, we present a comprehensive study on the subject. Five possible processes are identified: two of them (respectively called VE, $H E)$ measure errors along one axis, and the remaining three (respectively called $Y E, P E$, and $A E$ ) take into consideration errors along both axes. Since the axes and their corresponding errors may have different physical dimensions, a procedure is proposed to compensate for this difference so that all processes could express their answers in the same consistent dimensions. As usual, to avoid mutual cancellation, errors are squared or taken in their absolute value. The two cases are separately studied.

In the case of squared errors, the five processes are tested in many scenarios of experimental points, both analytically (using the software Mathematica) and numerically (with programs written on Python platform employing the Nelder-Mead optimization method). The investigation showed the possible existence of multiple solutions. Different answers originating from different starting points in NelderMead correspond to solutions revealed by analytic search with Mathematica. For each scenario of experimental points, it was found that the best lines of the five processes intercept at a common point. Furthermore, the point of intercept happens to coincide with the 'center of mass' of the scenario. This fact is described by stating the existence of an empirical 'Meeting Point Law'.

The case of absolute errors is only treated numerically, with NelderMead minimizer. As expected, the absolute error option shows greater robustness against outliers than the square error option, for all processes. The Meeting Point Law is not valid in this case.

By taking the value of minimized error as a criterion, the five processes are compared for efficiency. On average, processes $P E$ and AE, that consider errors along both axes, resulted in the smallest minimized error and may be considered the best processes. Processes that rely on errors along a single axis (VE, HE) stay at the second place. In all cases, YE is the process that results in the largest minimized errors.
\end{abstract}

Keywords: Linear data fitting, least squares, absolute deviation, analytical solution, numerical solution, Meeting Point Law, Nelder-Mead method, outliers, initial values. 


\section{Introduction}

Experimental points often result arranged in a cloud whose shape suggests a linear dependence between the two variables involved. The problem of drawing the 'best fitting line' through the experimental points is usually solved by minimizing the error arising from an assumed line and the experimental points. Different error criteria and different minimizing procedures give different 'best lines'

Figure 1.1 shows the position of a generic experimental point $P_{i}\left(s_{i}, t_{i}\right)$ relative to a proposed fitting line $t=x s+y$. Please note that the figure uses $(s, t)$ as coordinates axes in place of the usual $(x, y)$. This choice results to be useful later when dealing with minimizing procedures. Therefore, the true unknowns of the problem are ( $x=$ gradient or slope and $y=$ intercept) of the fitting line. Triangle $A_{i} P_{i} B_{i}$ is a right triangle with the right angle at vertex $P_{i}$.

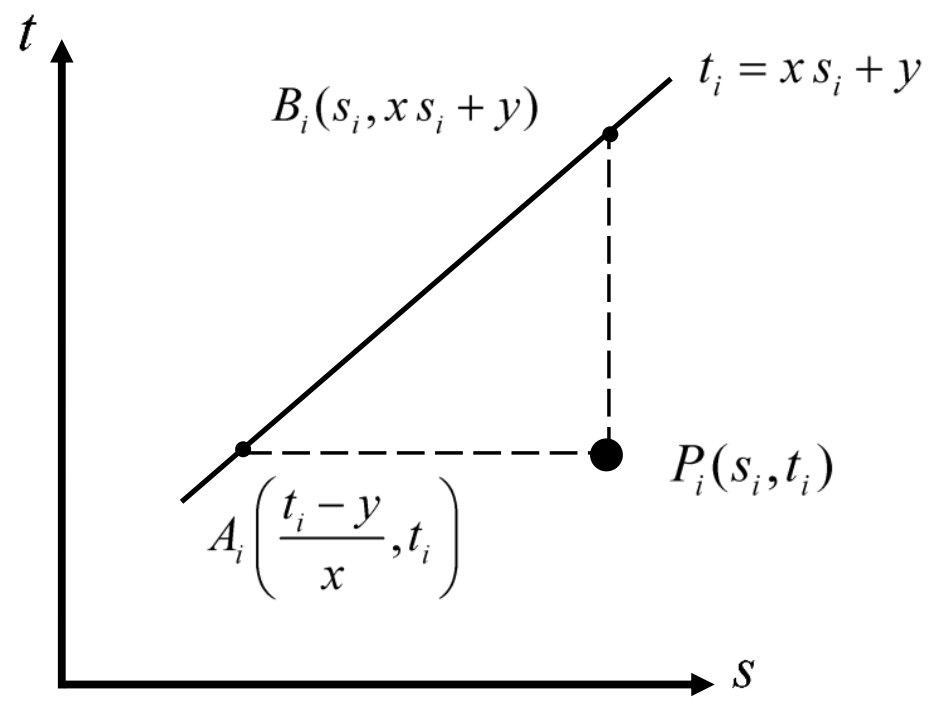

Figure 1.1 - A generic experimental point $P_{i}$ shown positioned in reference to proposed fitting line $t=x s+y$

The classical process to find a best line runs as follows: let us consider the distance $P_{i} B_{i}$ for each experimental point (an individual 'error'), square its value, sum for all points; let us name as $E^{2}$ the resulting total-squared-error. The best line is the line whose parameters ( $x$ = gradient, $y=$ intercept) minimize $E^{2}$.

This method has some advantages: squaring individual errors avoids mutual cancellation when positive and negative errors are summed; $E^{2}$ results to be a second-degree polynomial easily minimized with elementary Calculus in order to find $(x, y)$. Disadvantage: points far away from the line (called the 'outliers') give an exaggerated contribution to the determination of the best line. In fact, it may be said that each point attracts the line towards itself with a force proportional to the square of its distance from the line. 
The method just described attracted the attention of mathematicians like Gauss and Lagrange, in the eighteen-nineteen centuries, and may today be labeled as the 'classical process'. This method appears in all online calculators that offer to compute the best line. But why always take errors $P_{i} B_{i}$ and not, for example, errors along the horizontal axis $P_{i} A_{i}$ ? A different best line would be obtained, which deserves the same consideration as the previous one. And why not to take the absolute value of the error, instead of squaring it? Would it not be a more natural thing to do? Objection: the absolute function is not everywhere differentiable, precluding the use of Calculus to handle it. Answer to the objection: we live in the twentieth-first century, when numerical methods are available to minimize non-differentiable functions!

With such thoughts in mind, we wrote an earlier paper (Gambirasio, G. Comment on Linear Data-fitting Methods, OSF Preprints, May 15, 2019, doi:10.31219/osf.io/56w7k), beginning a discussion on different criteria for finding the best line. A more complete study is offered in the present paper.

Historically, the problem of the best fitting line has been treated from two different points of view: either a deterministic one or as a statistical question. We take the deterministic point of view here. We will not use regression or correlation in this study. In fact, we will not adopt any statistical point of view (whether the experimental points have random errors and what is their effect on the best line). Also, any classification between independent and dependent variables is considered irrelevant; it does not matter which variable is assigned to the $s$-axis (abscissas) and the other to the $t$-axis (ordinates).

Pearson's correlation coefficient, sometimes also computed here together with the best line, is not related to fitting lines. This coefficient is a characteristic of the distribution of points only. For example, taking errors horizontally gives a different fitting line from taking errors vertically, but the correlation coefficient is the same in both cases.

With reference to Figure 1.1, we propose to study five different ways (called here 'processes') to consider errors, divided in two categories: those that measure errors along one axis (processes VE and $\mathrm{HE}$ ), and those that take into consideration errors along both axes (processes $\mathrm{YE}, \mathrm{PE}$, and $\mathrm{AE}$ ), as follows:

1) process VE measures errors $P_{i} B_{i}$ along the vertical axis. When the errors are squared, VE becomes identical to the 'classical' process; when the absolute value of the errors are taken, VE leads to the so-called 'least absolute deviation method'.

2) process HE measures errors $A_{i} P_{i}$ along the horizontal axis.

3) process YE measures error $A_{i} B_{i}$ along the hypotenuse, thereby using values of errors from both axes.

4) process $\mathrm{PE}$ considers errors along the height of the triangle drawn from $P_{i}$ to the hypotenuse. It also makes use of errors from both axes.

5) process $\mathrm{AE}$ considers the area of triangle $A_{i} P_{i} B_{i}$ as the pertinent error, therefore using values of errors from both axes. Since the area is a length squared, for comparison purposes with the other processes whose errors are lengths, we take the square root of the area as representing process AE. 


\section{Different physical dimensions}

In real life, as shown in the example of Figure 2.1, the coordinates of experimental points have physical dimensions, as well as the best fitting line. This fact must be taken into consideration when conducting a study of different criteria for finding the line. For example, the classical method (errors taken along the $t$-axis) will give its answers in terms of units of $t$-axis. On the other hand, taking errors along the $s$-axis (a different criterion) will give its answers in terms of units of $s$-axis, which may be of different physical dimensions from the units of $t$-axis. In the example of Figure 2.1, volts are the units on $s$ axis and amperes on $t$-axis. Then, how to compare the two answers?

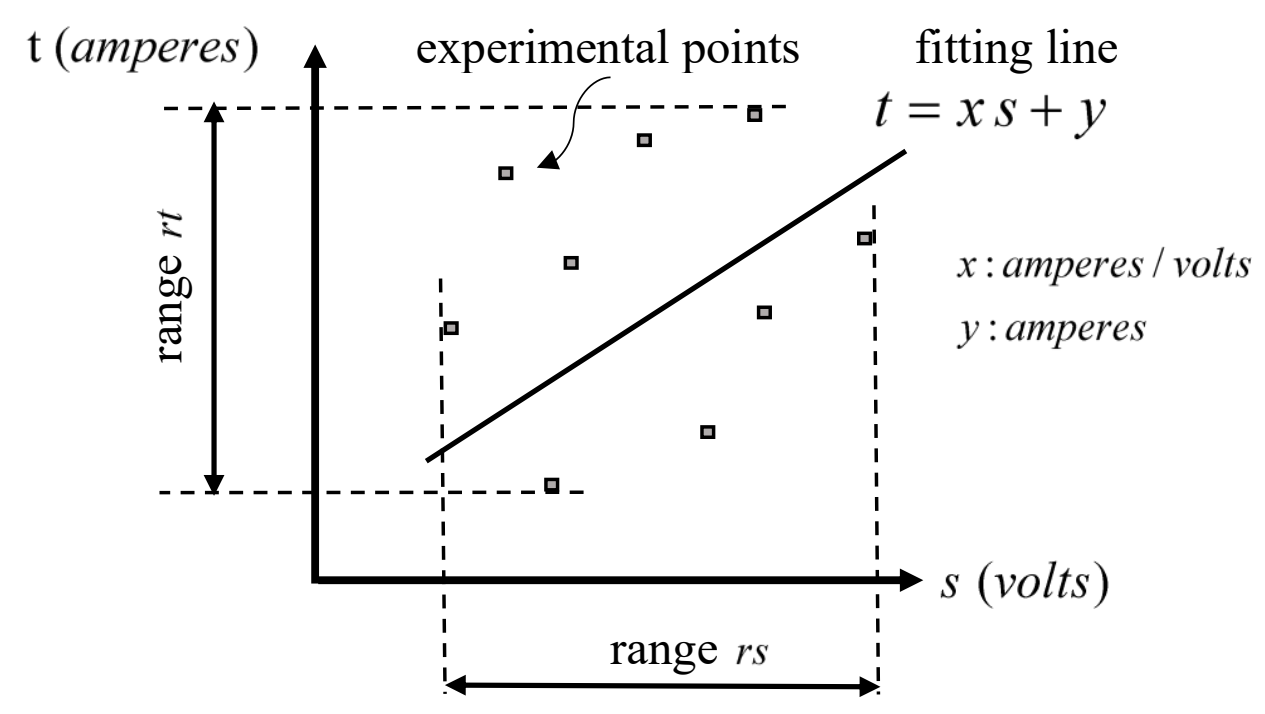

Figure 2.1 - In real life, data have physical dimensions

Another difficulty arises when the chosen criterion uses information from both axes. With reference to Figure 1.1, now consider process YE, which minimizes the sum of hypotenuses $A_{i} B_{i}$.

In the right triangle $A_{i} P_{i} B_{i}$, Pythagoras' Theorem gives $\left(A_{i} B_{i}\right)^{2}=\left(P_{i} A_{i}\right)^{2}+\left(P_{i} B_{i}\right)^{2}$.

But the theorem is about lengths, that is, $P_{i} A_{i}$ and $P_{i} B_{i}$ (and therefore $A_{i} B_{i}$ ) must all be expressed, for example, in centimeters. Or, at least, in the same physical dimension: all in volts, or all in amperes.

Therefore, all the sides of the triangle must be previously expressed in the same physical units, and then the theorem may be safely applied.

When the experimental points are plotted on a graph and we agree on using centimeters to measure distances on it, then automatically we apply some 'graphical conversion factors' like:

$$
\begin{aligned}
& \text { (centimeters/units of } s \text {-axis) }=c f s, \\
& \text { (centimeters/units of } t \text {-axis) }=c f t .
\end{aligned}
$$

Now Pythagoras' Theorem may be correctly written as 
[hypotenuse in centimeters $]^{2}=$

$=[(\text { horizontal short side in units of } s \text {-axis).(centimeters/units of } s \text {-axis })]^{2}+$

$+[(\text { vertical short side in units of } t \text {-axis).(centimeters/units of } t \text {-axis })]^{2}=$

$=[\text { (horizontal short side in units of } s \text {-axis). } c f s]^{2}+$

$+[\text { (vertical short side in units of } t \text {-axis). } c f t]^{2}$

If we wish to compute errors in units of $s$-axis, then the above equation must be divided by $(c f s)^{2}$ :

$$
\begin{gathered}
\text { [hypotenuse in units of } s \text {-axis }]^{2}= \\
=[(\text { hypotenuse in centimeters }) / c f s]^{2}= \\
=[\text { horizontal short side in units of } s \text {-axis }]^{2}+ \\
+[(\text { vertical short side in units of } t \text {-axis }) . c f t / c f s]^{2} .
\end{gathered}
$$

Let $K>0$ be a conversion factor defined as: $K=c f t / c f s$; then

[hypotenuse in units of $s$-axis $]^{2}=$

$=[$ (horizontal short side in units of $s$-axis $)]^{2}+$

$+[\text { (vertical short side in units of } t \text {-axis). } K]^{2}$.

Notice that (units of $K)=($ units of $s$-axis $) /($ units of $t$-axis $)$

Let us write a numerical example to make things clearer.

Referring to Figure 2.1, let us call 'range' a measure of how the experimental points spread (horizontally or vertically), then define $r s$ to be the range of points along $s$-axis, that is: $r s=[($ greatest value in $s)$ - (smallest value in $s)]$. Similarly, we define $r t$ to be the range of points along $t$-axis: $r t=$ [(greatest value in $t)$ - (smallest value in $t)]$. Suppose now that we use a plot sized to contain all experimental points, such as range $r s$ corresponds to a length $h$ in the $s$-axis, and range $r t$ corresponds to a length $v$ in the $t$-axis.

Then:

$$
\begin{aligned}
& \text { conversion factor for } s \text {-axis: } c f s=h / r s \text {; } \\
& \text { conversion factor for } t \text {-axis: } c f t=v / r t \text {; and } \\
& K=c f t / c f s=(v / r t) /(h / r s)=(v . r s) /(h . r t) .
\end{aligned}
$$

In our example, let $h=20 \mathrm{~cm}$ and $v=15 \mathrm{~cm}$. And let $r s=9.5 \mathrm{~V}$ and $r t=16 \mathrm{~A}$. Then

$$
\begin{gathered}
\text { (centimeters/units of } s \text {-axis })=h / r s=20 \mathrm{~cm} / 9.5 \mathrm{~V} ; \\
\text { (centimeters/units of } t \text {-axis })=v / r t=15 \mathrm{~cm} / 16 \mathrm{~A} ; \text { and } \\
K=(15 \times 9.5) /(20 \times 16)=0.445 \mathrm{~V} / \mathrm{A}
\end{gathered}
$$

This is the value of $K$ computed for a specific arrangement of plotting and experimental points. By using this value of $K$, the error is certainly expressed in units of $s$-axis (that is, in volts, in the case at hand). Whenever we use a value of $K$, we hypothetically assume a corresponding configuration of graph and of experimental points. 


\section{The five processes}

In this section we will establish the mathematical expressions of the five processes already defined.

In the study to follow, we will consider sets of experimental points in which the abscissas are always the same and the ordinates are changed to perform the study. Each such set is called a scenario. In order to get results that are comparable with one another, all results must be expressed in the same physical units (those of the $s$-axis, by our choice).

The set of $n$ experimental points (the 'scenario') is called $P$ :

$$
P \rightarrow\left(s_{i}, t_{i}\right) \quad, i=1,2, \ldots, n
$$

For the sake of generality, the errors are taken in their absolute values and elevated to an arbitrary exponent $p>0$. When $p=2$, we will be doing a 'least square' search for the best fitting line; when $p=1$, the 'absolute value' model will be followed. (Obviously, other values of $p$ can be chosen, if one wishes).

Then, let us write the sum of vertical errors $P_{i} B_{i}$ (as per Figure 1.1) in a general case in which the errors are taken in their absolute value, elevated to exponent $p>0$, and summed over the $n$ experimental points. The result is a function $S(x, y)$, which will be expressed simply as $S$ here:

$$
S=\sum_{i}\left|x s_{i}+y-t_{i}\right|^{p} \quad, i=1,2, \ldots, n
$$

The vertical-error process (VE process) is obtained by minimizing $S$ relatively to $x$ and $y$, respectively the gradient and the intercept of the best line: VE process $=\min _{x, y} S(x, y)$, expressed here as $\min \{S\}$.

The errors are now given in units of $(t)^{p}$, but since we want the results expressed in units of $(s)^{p}$, factor $K$ must be introduced:

$$
\mathrm{VE} \text { process }=\min \left\{K^{p} . S\right\}
$$

Let us now write an expression similar to $S$ but concerning the horizontal errors $P_{i} A_{i}$. Call it $S^{\prime}$, provisionally:

$$
S^{\prime}=\sum_{i}\left|\left(t_{i}-y\right) x^{-1}-s_{i}\right|^{p}=\sum_{i}\left|\left(t_{i}-y-x s_{i}\right) / x\right|^{p}, i=1,2, \ldots, n
$$

We may now obtain the best fitting line according to the HE process (the horizontal-error process) by minimizing $S^{\prime}$ relatively to $x$ and $y$. But this means minimizing $\left[|x|^{-p} \cdot S\right]$, and the minimum error results expressed in units of $(s)^{p}$ :

$$
\text { HE process }=\min \left\{|x|^{-p} \cdot S\right\}
$$

without factor $K$. 
We now choose, as a new process, one about which we already wrote extensively: the YE process (the hypotenuse process), in which the sum of hypotenuses $A_{i} B_{i}$ is to be minimized.

In right triangle $A_{i} P_{i} B_{i}$, Pythagoras' Theorem gives $\left(A_{i} B_{i}\right)^{2}=\left(P_{i} A_{i}\right)^{2}+\left(P_{i} B_{i}\right)^{2}$. In accordance with what was concluded in the previous discussion, the hypotenuse relative to point $P_{i}$ is expressed in:

$$
\left(\text { hypotenuse }_{i}\right)^{2}=\left[K .\left(x . s_{i}+y-t_{i}\right)\right]^{2}+\left\{\left[s_{i}-\left(t_{i}-y\right) / x\right]\right\}^{2}=\left(x . s_{i}+y-t_{i}\right)^{2} \cdot\left[K^{2}+x^{-2}\right]
$$

and taking the positive solution of the square root:

$$
\text { hypotenuse }_{i}=\left(x \cdot s_{i}+y-t_{i}\right) \cdot\left[K^{2}+x^{-2}\right]^{1 / 2}
$$

After taking absolute values, writing exponent $p$ and summing for $i=1,2, \ldots, n$ we finally get

$$
\text { YE process }=\min \left\{\left|\left(K^{2}+x^{-2}\right)\right|^{p / 2} \cdot S\right\}
$$

The next process, PE process, considers the height (perpendicular distance from the right angle to the line) as the relevant element.

The height of a right triangle, as defined above, may be calculated by the following identity:

$$
\text { height }=(\text { first short side }) .(\text { second short side }) /(\text { hypotenuse }),
$$

all sides dutifully expressed in the same physical dimension $(s)^{p}$. For a generic point $P_{i}$ the expression is given by:

$$
\begin{gathered}
\text { height }_{i}=\left[K\left(x . s_{i}+y-t_{i}\right)(1 / x)\left(x . s_{i}+y-t_{i}\right)\right] /\left[ \pm\left(x \cdot s_{i}+y-t_{i}\right) \cdot\left(K^{2}+x^{-2}\right)^{1 / 2}\right]= \\
= \pm K\left(x . s_{i}+y-t_{i}\right)\left(x^{2} K^{2}+1\right)^{-1 / 2}
\end{gathered}
$$

Taking absolute values, adding exponent $p$, and summing over all points, the result is:

$$
\mathrm{PE} \text { process }=\min \left\{\left|\left[K\left(x^{2} K^{2}+1\right)^{-1 / 2}\right]\right|^{p} . S\right\}
$$

The next, last process, $\mathbf{A E}$ process, consists of taking the area of triangle $A_{i} P_{i} B_{i}$ as the relevant element in evaluating errors. However, since the minimum error would be of higher dimension than the errors given by the other processes, we instead take the square root of the area. The resulting process AE is expected to express errors in units of $(s)^{p}$. For each experimental point $P_{i}$, we have:

$$
\begin{gathered}
\mid \text { area }\left._{i}\right|^{1 / 2}=\left|(1 / 2) .\left(P_{i} B_{i}\right)\left(P_{i} A_{i}\right)\right|^{1 / 2}=\left|(1 / 2) K\left(x . s_{i}+y-t_{i}\right)(1 / x)\left(x . s_{i}+y-t_{i}\right)\right|^{1 / 2}= \\
=|K /(2 x)|^{1 / 2} \cdot\left|\left(x . s_{i}+y-t_{i}\right)\right|
\end{gathered}
$$


Taking absolute values, adding exponent $p$, and summing over all points, we get:

$$
\mathrm{AE} \text { process }=\min \left\{|K /(2 x)|^{p / 2} \cdot S\right\}
$$

\section{Gallery of processes}

All five processes are summarized here. Reminding that core sum $S$ was defined as:

$$
S=\sum_{i}\left|x s_{i}+y-t_{i}\right|^{p} \quad, i=1,2, \ldots, n \quad,
$$

it is apparent, from expressions (1) to (5) that for all different processes we always end up with an expression that depends on this core sum and a factor:

$$
\text { process }=\min \{(\text { a factor depending of the process }) . S\}
$$

This fact is useful from the point of view of computation, because the calculation of $S$ (a characteristic of the experimental points distribution) can be programmed in a common block, and the factor specific of each process is added at the time of minimization. This factor that depends on each process is called $F$.

The factors $F$ for the five processes are listed in Table 4.1 and the process errors are visually summarized in Figure 4.1 

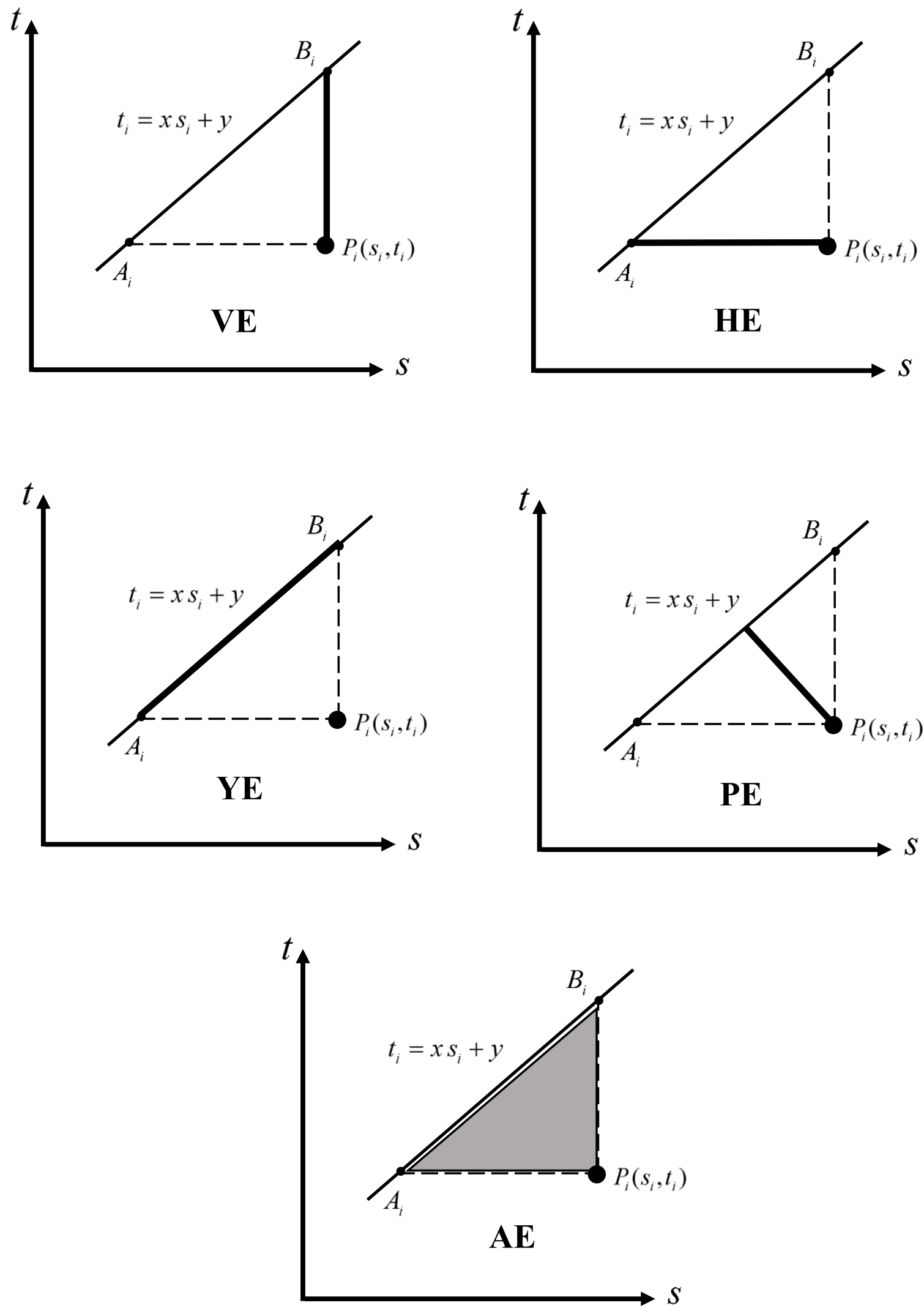

Figure 4.1 - The five processes for error definition 
Table 4.1 - Minimizing functions and Factor $F$ for the five processes

\begin{tabular}{|c|c|c|}
\hline Process & $\begin{array}{l}\text { Minimizing function } \\
\quad \text { (in }(s)^{p} \text { units) }\end{array}$ & Factor $F$ \\
\hline VE (vertical) & $\min \left\{K^{p} . S\right\}$ & $K^{p}$ \\
\hline HE (horizontal) & $\min \left\{|x|^{-p} . S\right\}$ & $|x|^{-p}$ \\
\hline YE (hypotenuse) & $\min \left\{\left(K^{2}+x^{-2}\right)^{p / 2} \cdot S\right\}$ & $\left(K^{2}+x^{-2}\right)^{p / 2}$ \\
\hline PE (height) & $\min \left\{\left[K\left(1+K^{2} x^{2}\right)^{-1 / 2}\right]^{p} \cdot S\right.$ & $\left.K\left(1+K^{2} x^{2}\right)^{-1 / 2}\right]^{p}$ \\
\hline $\mathrm{AE}$ (sqr area) & $\min \left\{\left|\left(\frac{K}{2 x}\right)\right|^{p / 2} . S\right.$ & $\left.\left(\frac{K}{2 x}\right)\right|^{p / 2}$ \\
\hline
\end{tabular}

\section{Choices}

There are many parameters in the expressions presented above and we must now discuss how to choose them.

In practical cases, only two values of $p$ are of interest: $p=2$ (as used in the classical case of least squares) and $p=1$ (a more natural approach to the problem of finding the best fitting line).

Establishing certain basic or 'normal' objects as scenarios to be used as references will help in organizing the research. The following set $P$ of 13 experimental points $\left(s_{i}, t_{i}\right)$ is a basic set for our study:

$P=\left\{\left[1,5 \times 10^{\mathrm{q}}\right],\left[2,6 \times 10^{\mathrm{q}}\right],\left[3,4 \times 10^{\mathrm{q}}\right],\left[4,4 \times 10^{\mathrm{q}}\right],\left[5,10 \times 10^{\mathrm{q}}\right],\left[6,12 \times 10^{\mathrm{q}}\right],\left[-2.5,2 \times 10^{\mathrm{q}}\right]\right.$, $\left.\left[-1.5,-1 \times 10^{\mathrm{q}}\right],\left[0.0,4.5 \times 10^{\mathrm{q}}\right],\left[2.3,0 \times 10^{\mathrm{q}}\right],[5.2,9 \times 10],\left[6.5,10 \times 10^{\mathrm{q}}\right],\left[7,15 \times 10^{\mathrm{q}}\right]\right\}$.

Each value of exponent $q$ defines a different scenario. In this work, values of $q$ are taken from the set $(-2,0,2)$.

A scenario is called 'normal' when exponent $q$ takes the value $q=0$ :

In the discussion over factor $K$ (section 2), a 'normal' size of graphic was arbitrarily selected as: $h=$ length of horizontal axis range $(s$-axis $)=20 \mathrm{~cm} ; v=$ length of vertical axis range $(t$-axis $)=15 \mathrm{~cm}$. It is supposed that experimental points and fitting lines are plotted there. Also, the values of ranges $r s$ and $r t$ used in section 2 are taken from the 'normal' set. In the 'normal' scenario, value of $K$ results as $K=0.445$.

We should emphasize the importance of factor $K$ in the expressions shown in Table 4.1. If $K$ was not present in the calculations of the minimizing functions, each process would result in values with different units. For example, in the case mentioned in section 2, with volts on $s$-axis and amperes on $t$-axis, errors from VE would result expressed in 
(amperes) $)^{p}$; errors from HE would be expressed in (volts) ${ }^{p}$; errors from YE would be expressed in $\left[(\text { volts })^{2}+(\text { amperes })^{2}\right]^{p / 2}$; errors from PE would result expressed in

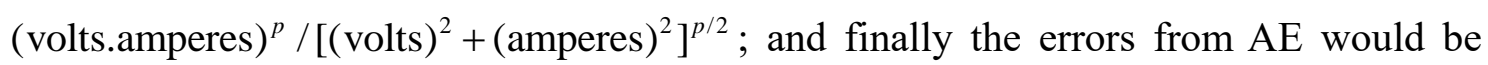
expressed as (volts.amperes) $)^{p / 2}$. Considering factor $K$, which is given in (volts/amperes) units, all the five minimizing functions are expressed in (volts) ${ }^{p}$ units, thus can be properly compared.

As seen from the above definition of $P$, the set of abscissas ( $s$-axis) is always the same; values of ordinates ( $t$-axis) change depending on the assumed $q$. The choice of keeping the same abscissas for all scenarios gives uniformity and better organization to the study. Programs in Python platform (https://www.python.org/) have been written to help in the mathematical computations and in plotting results. Python offers 14 methods for minimizing functions, not all useful in all situations and for our purposes. Using absolute values when evaluating errors turns the functions non-differentiable in certain points, thus making the choice of a good method a very critical factor. For a better organization of the paper, the case of $p=2$ is presented first and separately from the case of $p=1$.

\section{Results for $p=2, q=0$ ('normal scenario', $K=0.445$ )}

Scenario: $P=\{[1,5],[2,6],[3,4],[4,4],[5,10],[6,12],[-2.5,2],[-1.5,-1],[0,4.5]$, $[2.3,0],[5.2,9],[6.5,10],[7,15]\}$

Table 6.1 summarizes the results obtained when exponent $p=2$ and $q=0$, with the scenario shown above. The minimized errors, expressed in $s^{2}$ units, suggest the better performance of $\mathrm{PE}$ and $\mathrm{AE}$ processes (smallest errors). Best fitting lines equations are also shown on the table, and their graphs are plotted in Figure 6.1.

Table 6.1 - Results for $p=2, q=0, K=0.445$

\begin{tabular}{|c|c|c|c|c|c|}
\hline Process & VE & HE & YE & PE & AE \\
\hline $\begin{array}{c}\text { Minimized } \\
\text { error value }\end{array}$ & 16.27 & 34.07 & 54.41 & 11.86 & 12.84 \\
\hline $\begin{array}{l}\text { Fitting line } \\
t=x . s+y\end{array}$ & $t=1.29 s+2.41$ & $t=1.86 s+0.75$ & $t=1.63 s+1.42$ & $t=1.45 s+1.96$ & $t=1.55 s+1.66$ \\
\hline
\end{tabular}




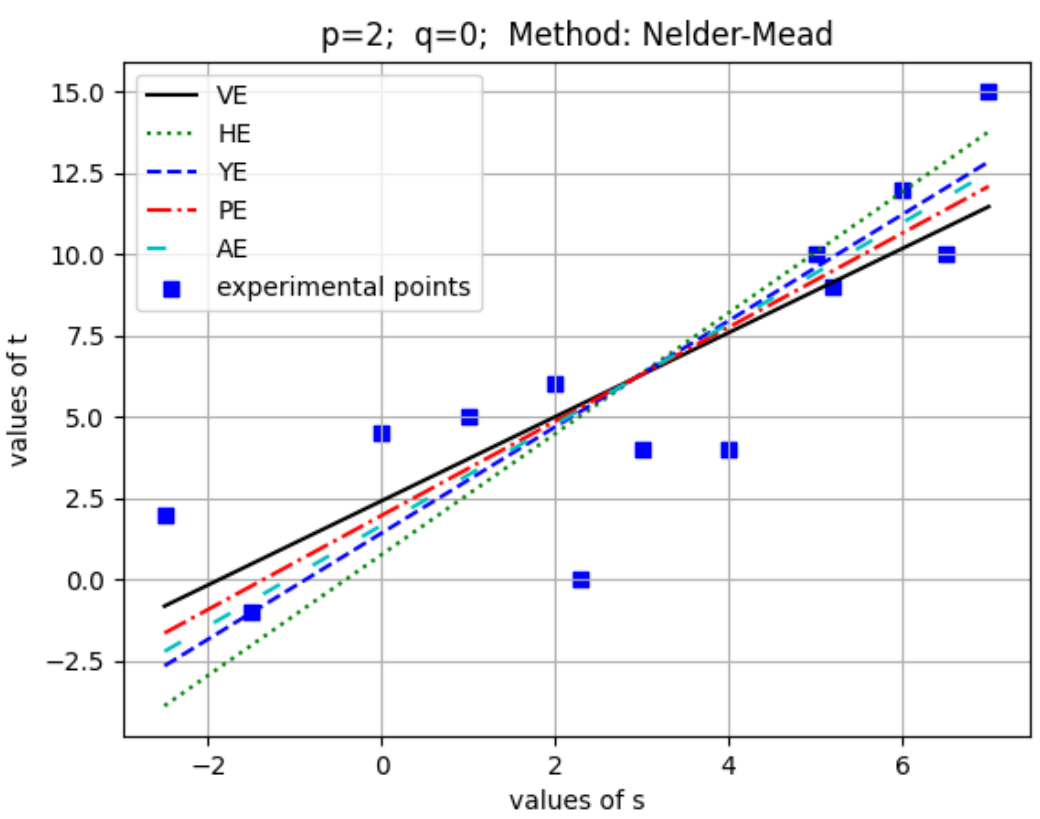

Fig. 6.1 - Best fitting lines corresponding to all five processes for $p=2, q=0, K=0.445$

\section{Results for $p=2, q=2, K=0.00445$}

The ordinates of experimental points are now one hundred times greater than in the preceding case, but the abscissas are always the same. The scenario is then:

$P=\{[1,500],[2,600],[3,400],[4,400],[5,1000],[6,1200],[-2.5,200],[-1.5,-100]$, $[0.0,450],[2.3,0],[5.2,900],[6.5,1000],[7,1500]\}$

The parameters (gradient and intercept) of lines resulted also one hundred times greater than in the previous case. However, the errors are the same as before, thus still showing that PE and AE are the better processes. All such information is in Table 7.1 and Figure 7.1 shows the corresponding fitting lines.

Table 7.1 - Results for $p=2, q=2, K=0.00445$

\begin{tabular}{|c|c|c|c|c|c|}
\hline Process & VE & HE & YE & PE & AE \\
\hline $\begin{array}{c}\text { Minimized } \\
\text { error value }\end{array}$ & 16.27 & 34.07 & 54.41 & 11.86 & 12.84 \\
\hline $\begin{array}{c}\text { Fitting line } \\
t=x . s+y\end{array}$ & $t=129 s+240$ & $t=186 s+75$ & $t=163 s+142$ & $t=145 s+196$ & $t=155 s+166$ \\
\hline
\end{tabular}




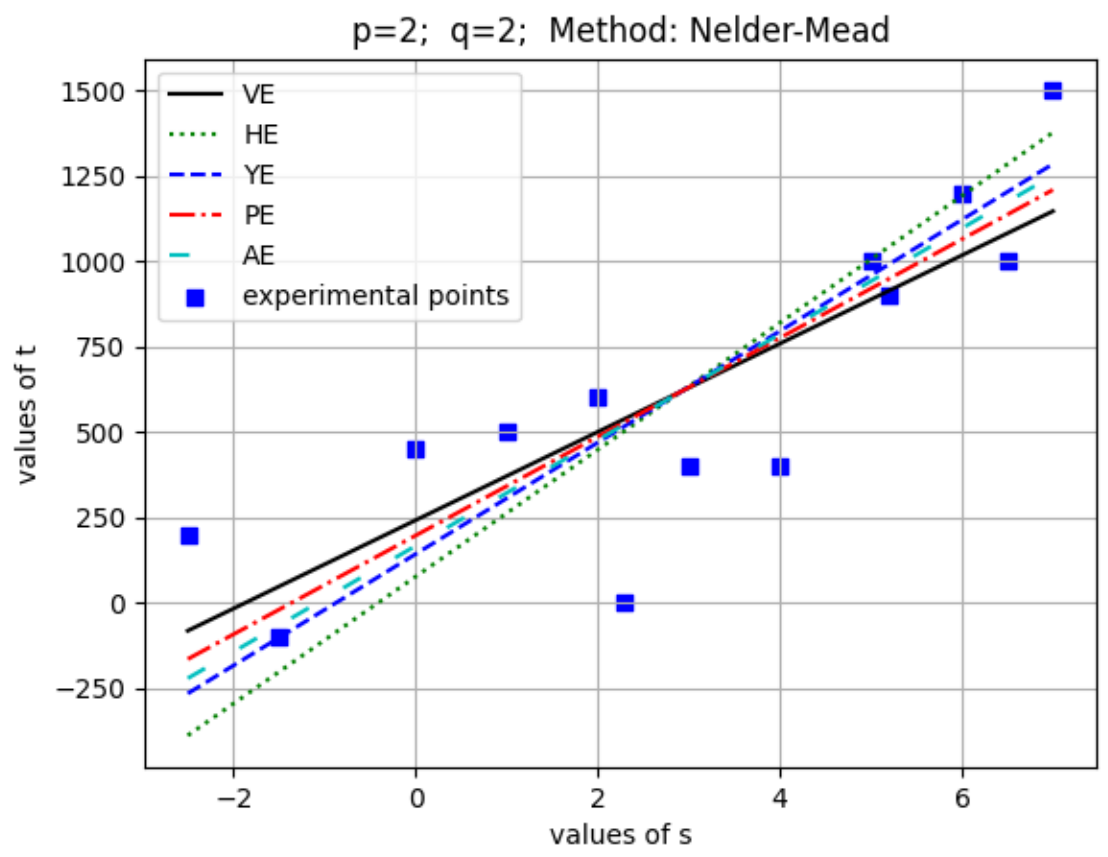

Fig. 7.1 - Best fitting lines corresponding to all five processes for $p=2, q=2, K=0.00445$

\section{Results for $p=2, q=-2, K=44.53$}

The ordinates of experimental points are now one hundred times smaller than in the case of section 6 ('normal' scenario), but the abscissas are always the same. The scenario is then:

$P=\{[1,1 / 20],[2,3 / 50],[3,1 / 25],[4,1 / 25],[5,1 / 10],[6,3 / 25],[-2.5,1 / 50],[-1.5,-$ $1 / 100],[0.0,0.045],[2.3,0],[5.2,9 / 100],[6.5,1 / 10],[7,3 / 20]\}$.

Table 8.1 shows the errors (same values as before) and the lines equations; the lines themselves are plotted in Figure 8.1.

Table 8.1 - Results for $p=2, q=-2 ; K=44.53$

\begin{tabular}{|c|c|c|c|c|c|}
\hline Process & VE & HE & YE & PE & AE \\
\hline $\begin{array}{l}\text { Minimized } \\
\text { error value }\end{array}$ & 16.27 & 34.07 & 54.41 & 11.86 & 12.84 \\
\hline $\begin{array}{c}\text { Fitting line } \\
t=x . s+y\end{array}$ & $t=0.013 s+0.024$ & $t=0.0186 s+0.0075$ & $t=0.016 s+0.014$ & $t=0.014 s+0.020$ & $t=0.016 s+0.017$ \\
\hline
\end{tabular}




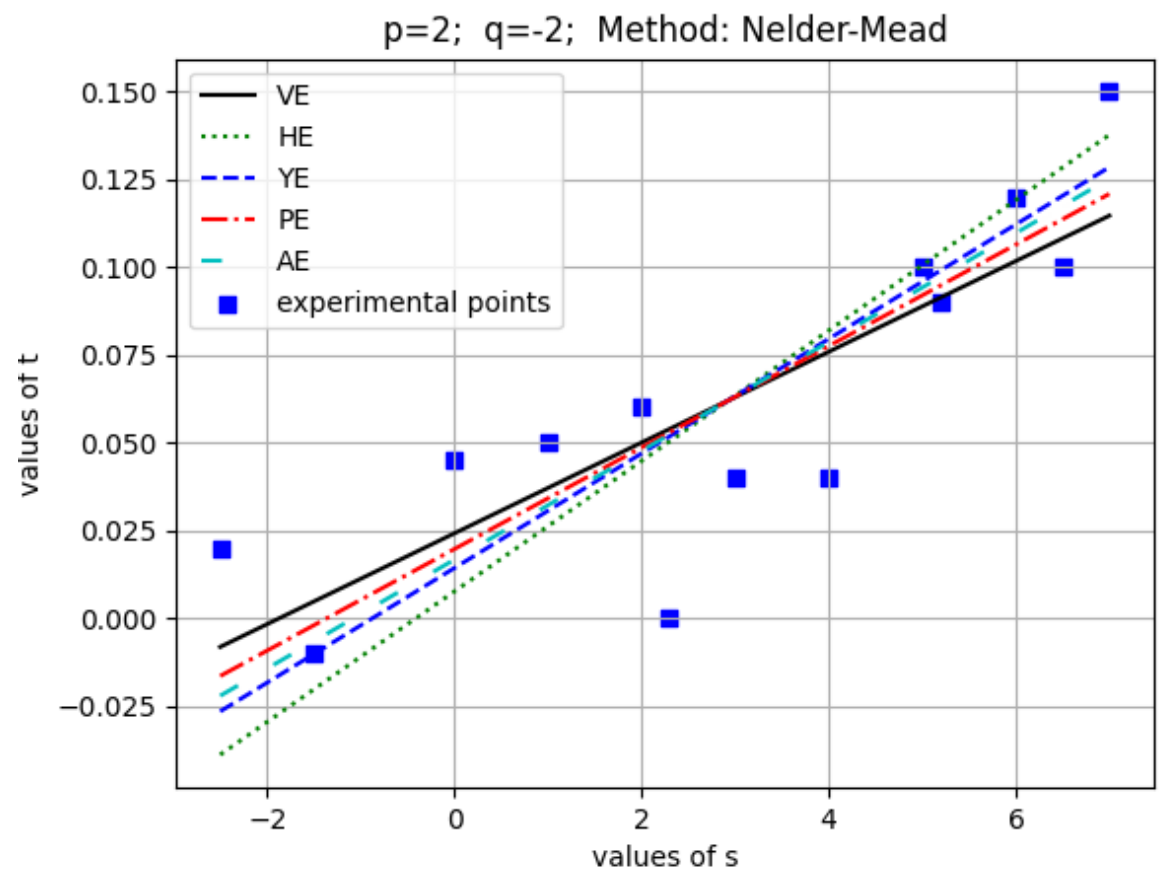

Fig. 8.1 - Best fitting lines corresponding to all five processes for $p=2, q=-2 ; K=44.53$

\section{The case with outliers}

In this section we shall examine a scenario with outliers, by changing the ordinates of two points in our normal set of points to higher values. For the sake of brevity, we will only show the results of the case $p=2, q=0$. Cases with $q=2$ and $q=-2$ follow the patterns shown previously and will bring no novel information.

The results of this section will be used later in section 13 , when $p=1$ and $p=2$ cases are compared for robustness against outliers. The scenario now is:

$P=\{[1,5],[2,6],[3,4],[\mathbf{4}, \mathbf{4 0}],[5,10],[6,12],[-2.5,2],[-1.5,-1],[\mathbf{0}, \mathbf{4 5}],[2.3,0],[5.2$, $9],[6.5,10],[7,15]\}$.

The two changed points are the ones with abscissa 4 and abscissa 0 , marked above in bold font. With this scenario, considering the same values of $h=20 \mathrm{~cm}$ and $v=15 \mathrm{~cm}$, and calculating the ranges $r s$ and $r t$ according to the set of experimental points, factor $K$ takes the value $K=0.155$.

Table 9.1 summarizes the results. In general, the minimized errors increase as compared with previous cases without outliers, and VE displaced AE as the second-best process. Why so? Because being all outliers located on the vertical, processes that take errors along the two axes are at disadvantage as compared with processes like VE that only concern the vertical direction. Conversely, process HE, that only considers errors along the horizontal axis, gives results that look strange in the new scenario, because nothing has changed in the horizontal direction. This last fact is dramatically shown in Figure 9.1. For greater clarity, Figure 9.2 shows the same lines except the line corresponding to HE. 
Table 9.1 - Results for $p=2, q=0$ with outliers; $K=0.155$

\begin{tabular}{|c|c|c|c|c|c|}
\hline Process & VE & HE & YE & PE & AE \\
\hline $\begin{array}{c}\text { Minimized } \\
\text { error value }\end{array}$ & 58 & 110 & 292 & 57 & 71 \\
\hline $\begin{array}{c}\text { Fitting line } \\
t=x . s+y\end{array}$ & $t=0.58 s+10.37$ & $t=37 s-97$ & $t=5.44 s-3.83$ & $t=1.19 s+8.60$ & $t=4.70 s+1.62$ \\
\hline
\end{tabular}

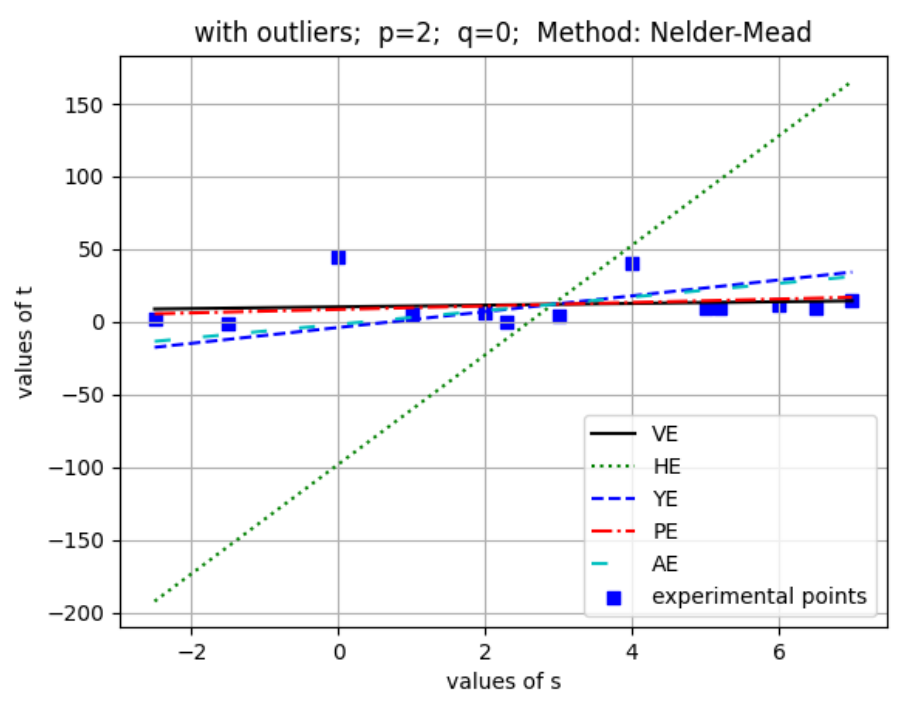

Fig. 9.1 - Best fitting lines corresponding to all five processes, for $p=2, q=0$ with outliers; $K=0.155$

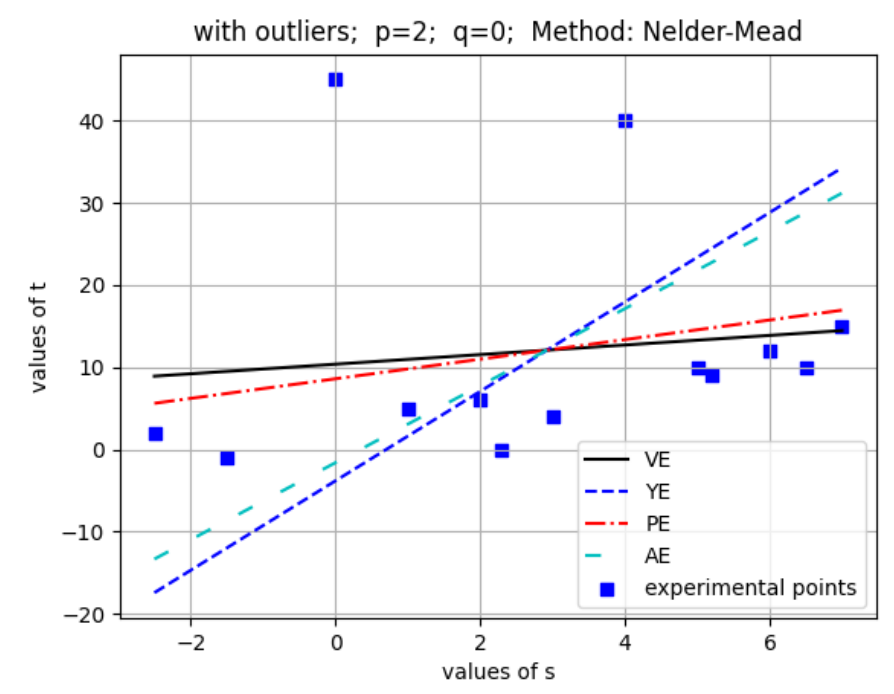

Fig. 9.2 - Same as Figure 9.1 but omitting the line corresponding to HE process

\section{Comparison of errors for $p=2, q=0$}

Table 10.1 summarizes the minimized value attained through each process, when using a Nelder-Mead method for minimizing the error function. It may be used to choose the 'best' 
process when $p=2$. Processes $\mathrm{PE}$ and $\mathrm{AE}$, that consider errors along the two axes, are strong candidates to be considered as the best processes, AE being replaced by VE when outliers in the $t$-axis are present within experimental points. Strangely, YE stands out as the worst process; it seems that the hypotenuse is not a good way to consider errors.

Table 10.1 - Comparison of minimized error for different processes for $p=2, q=0$

\begin{tabular}{|c|c|c|c|c|c|}
\hline Process & VE & HE & YE & PE & AE \\
\hline $\begin{array}{c}\text { normal } \\
\text { scenario } \\
(K=0.445)\end{array}$ & 16 & 34 & 54 & 12 & 13 \\
\hline $\begin{array}{c}\text { scenario with } \\
\text { outliers } \\
(K=0.155)\end{array}$ & 58 & 110 & 292 & 57 & 71 \\
\hline
\end{tabular}

\section{The Meeting Point Law}

\subsection{Introduction}

The reader has undoubtedly noticed that, in the preceding graphs, all the lines seem to intercept at one point. That is, there is a 'meeting point' common to the lines of all processes in a scenario. We intend to study such curious behavior in this section.

The behavior becomes 'curiouser and curiouser' when we further discover that the 'meeting point' is the center of mass of the experimental points! Rather presumptuously, we call the finding 'The Meeting Point Law'.

It may be argued that all this may be a coincidence. With a different scenario, things could be different. We shall now analytically study whether the proposed Law holds for all processes. If it does, then we can conclude that the Law is independent of scenarios.

First, we find the center of mass by summing over the $n$ experimental points $\left(s_{i}, t_{i}\right)$ :

$$
\begin{gathered}
\text { abscissa of center of mass } s_{C M}=\left(\sum_{i} s_{i}\right) / n, \\
\text { ordinate of center of mass } t_{C M}=\left(\sum_{i} t_{i}\right) / n
\end{gathered}
$$

Second, for each line $t=x \cdot s+y$ we make $s=s_{C M}$ and calculate $t_{c r}$ as:

$$
t_{c r}=x \cdot s_{C M}+y
$$

According to the proposed Law, $t_{c r}$ should coincide with $t_{C M}$. We will check it analytically so that the conclusion will not depend on any particular scenario but, rather, will be valid for all scenarios. Of course, at this moment, only for $p=2$. What happens when $p=1$ we will see later on. 
As from discussions in section 4, the generic expression $Q$ of the error to be minimized is written for $p=2$ as:

$$
\begin{gathered}
Q=F \cdot \sum_{i}\left(x s_{i}+y-t_{i}\right)^{2} \quad, i=1,2, \ldots, n \\
\text { or: } \\
Q=F\left[\left(x^{2}\right) \sum_{i}\left(s_{i}\right)^{2}+n y^{2}+\sum_{i}\left(t_{i}\right)^{2}+2 x y \sum_{i} s_{i}-2 x \sum_{i} s_{i} t_{i}-2 y \sum_{i} t_{i}\right]
\end{gathered}
$$

where the factors $F$ are given in Table 11.1 for the five processes.

Notice that for process AE the absolute value of $x$ must be kept, in order to guarantee for no error cancellation. Therefore, the minimizing function becomes non-differentiable at certain points, and it is not possible to perform an analytic study of this process.

Table 11.1 - Factor $F$ for $p=2$

\begin{tabular}{|c|c|}
\hline Process & Factor $\boldsymbol{F}$ \\
\hline VE (vertical) & $K^{2}$ \\
\hline HE (horizontal) & $x^{-2}$ \\
\hline YE (hypotenuse) & $K^{2}+x^{-2}$ \\
\hline PE (height) & $K^{2} /\left(1+K^{2} x^{2}\right)$ \\
\hline AE (sqr of area) & $\frac{K}{2|x|}$ \\
\hline
\end{tabular}

For each process, except $\mathrm{AE}$, the values of $x$ and $y$ are found by minimizing $Q$, solving the following system of two partial derivatives:

$$
\left\{\begin{array}{l}
\frac{\partial Q}{\partial x}=0 \\
\frac{\partial Q}{\partial y}=0
\end{array}\right.
$$

These systems have been solved using software Mathematica (Wolfram Mathematica: https://www.wolfram.com/mathematica/ ). Sometimes there are surprises: more than one solution appears; some solutions are complex, that is, $x$ or/and $y$ are complex numbers. Also, with the help of Mathematica, for each solution it has been checked whether $t_{c r}=t_{C M}$, whereby confirming, or not, the proposed Law of Meeting Point for each case at hand. This checking is made analytically, not numerically, therefore, the conclusions should not depend on any particular scenario. Unfortunately, for process AE we can only 
obtain numerical solutions.

To graphically show the fitting lines corresponding to the solutions, Mathematica also was used to numerically perform all computations. For this, we needed to put numbers into the parameters, using the 'normal scenario' $(q=0)$ of experimental points, as described in section 6 .

Constants and parameters used to prepare the graphs for this scenario are:

$K=0.445 ; n=13 ; s_{C M}=2.92 ; t_{C M}=6.19$;

$\sum_{i}\left(s_{i}\right)^{2}=223.08 ; \sum_{i}\left(t_{i}\right)^{2}=768.25 ; \sum_{i} s_{i}=38.0 ; \sum_{i} t_{i}=80.5 ; \sum_{i} s_{i} t_{i}=380.3$.

When solutions (or lines) are complex (in the form: $t=(a+j b) s+(c+j d)$ ), the corresponding graphs must be three-dimensional.

Complex lines come in pairs: a line and its conjugate. Every line intercepts its conjugate at the real point $(-d / b,(c b-a d) / b)$, the only real point in both lines. Therefore, the checking of $t_{c r}=t_{C M}$ is actually performed on the real plane of the graph.

We will now describe the results for each process, considering our 'normal scenario' of experimental points.

\subsection{Process VE}

Process VE is the old, time-honored process. Analytically, the values of $x$ and $y$ are found to be:

$$
\begin{gathered}
x=\left(\sum_{i} s_{i} t_{i}-t_{C M} \sum_{i} s_{i}\right) /\left(\sum_{i} s_{i}^{2}-s_{C M} \sum_{i} s_{i}\right) \\
y=\left(t_{C M} \cdot \sum_{i} s_{i}^{2}-s_{C M} \sum_{i} s_{i} t_{i}\right) /\left(\sum_{i} s_{i}^{2}-s_{C M} \sum_{i} s_{i}\right)
\end{gathered}
$$

and $t_{c r}=x \cdot s_{C M}+y=t_{C M}$.

Therefore, analytically, process VE only has one solution $(x, y)$ and follows the Meeting Point Law.

Numerically, in our normal scenario the result is $(x=1.29, y=2.41)$.

These are the same values obtained by optimization (shown in Table 6.1), using the Nelder-Mead method, verified in the range of initial values from $(-100,2)$ to $(+100,2)$.

\subsection{Process HE}

Analytically, the values of $x$ and $y$ are calculated as:

$$
\begin{gathered}
x=\left(n \cdot t_{C M}^{2}-\sum_{i} t_{i}^{2}\right) /\left(n \cdot s_{C M} \cdot t_{C M}-\sum_{i} s_{i} t_{i}\right) \\
y=\left(s_{C M} \sum_{i} t_{i}^{2}-t_{C M} \sum_{i} s_{i} t_{i}\right) /\left(n \cdot s_{C M} \cdot t_{C M}-\sum_{i} s_{i} t_{i}\right)
\end{gathered}
$$

and $t_{c r}=x \cdot s_{C M}+y=t_{C M}$. 
Process HE shows a similar situation as before: one analytic solution, and obedience to the Law, with numerical solution $(x=1.86, y=0.75)$ calculated by Mathematica.

With method Nelder-Mead, however, there appears a second solution when the initial value of $x$ goes negative. This second solution gives a negative $x$ in order of magnitude of $-10^{16}$ and a positive $y$ in order of magnitude of $10^{16}$. The meaning of this result is that the method is not able to converge for negative initial values of $\mathrm{x}$.

Figure 11.3 shows how $x$ and $y$ behave when the initial $x$ varies in the range $(-5,5)$; for the sake of clarity, highest values of parameters have been limited to $x=-8$ and $y=7$.

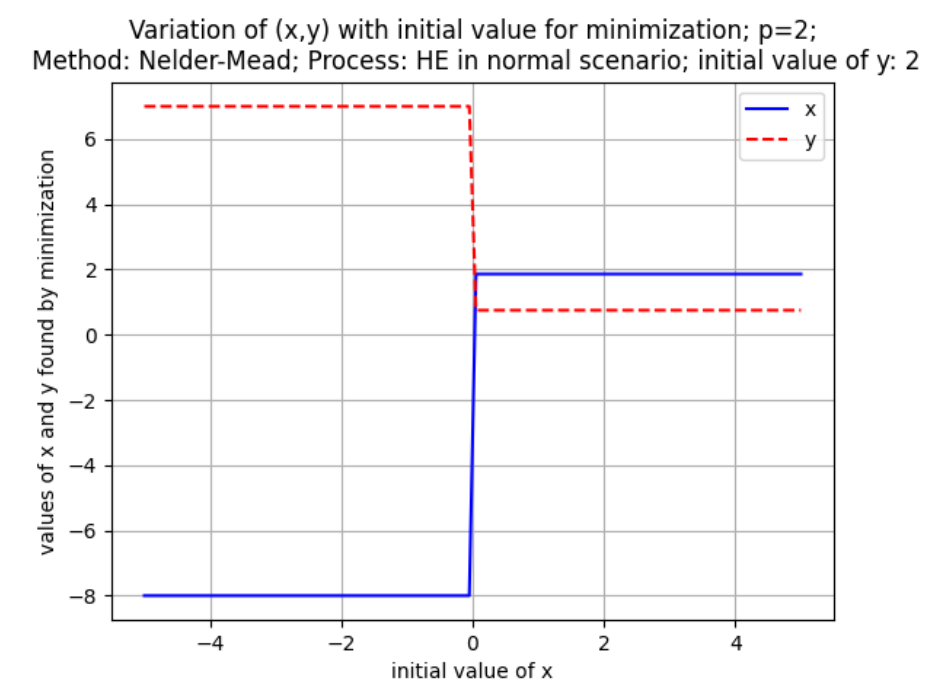

Fig. 11.3 - Process HE: change of values in $(x, y)$ in response to changes in the initial values, by a Nelder-Mead minimization, in a normal scenario

\subsection{Process YE}

Process YE shows a remarkable behavior: not less than eight solutions have been found analytically! We do not show the mathematical expressions for the analytical solutions here; some expressions are so long that it would take a few pages to print all of them.

The numerical values of such solutions have been computed with Mathematica using the experimental points of the 'normal' scenario, and the results are summarized in Table 11.4. Only two solutions ( 5 and 6 ) resulted in real parameters for $(x, y)$. However, the condition imposed by the Meeting Point Law, that is $t_{c r}=t_{C M}$, is attended by these real solutions, as well as by two complex solutions ( 7 and 8 ), as indicated by the $t_{c r}$ in bold characters in the table and in graph of Figure 11.4.1 and Figure 11.4.2.

Graphics of Figures 11.4.1 to 11.4.3 illustrate the positions of some lines that represent the analytical solutions. The abbreviated names accompany the solutions: $t 1$ means solution 1 , etc. 
Table 11.4 - Summary of numerical results for YE

\begin{tabular}{|c|c|c|c|}
\hline Solution & $x$ & $y$ & $t_{c r}$ \\
\hline 1 & $-2.25 \mathrm{j}$ & $12.42+2.55 \mathrm{j}$ & $12.42-4.02 \mathrm{j}$ \\
\hline 2 & $-2.25 \mathrm{j}$ & $-0.04+10.58 \mathrm{j}$ & $-0.04+4.02 \mathrm{j}$ \\
\hline 3 & $2.25 \mathrm{j}$ & $12.42-2.55 \mathrm{j}$ & $12.42+4.02 \mathrm{j}$ \\
\hline 4 & $2.25 \mathrm{j}$ & $-0.04-10.58 \mathrm{j}$ & $-0.04-4.02 \mathrm{j}$ \\
\hline 5 & -1.97 & 11.95 & $\mathbf{6 . 1 9}$ \\
\hline 6 & 1.63 & 1.42 & $\mathbf{6 . 1 9}$ \\
\hline 7 & $0.82-1.76 \mathrm{j}$ & $3.81+5.15 \mathrm{j}$ & $\mathbf{6 . 1 9}$ \\
\hline 8 & $0.82+1.76 \mathrm{j}$ & $3.81-5.15 \mathrm{j}$ & $\mathbf{6 . 1 9}$ \\
\hline
\end{tabular}

Plot of complex and real lines, with the real $(z=0)$ plane in transparent grey

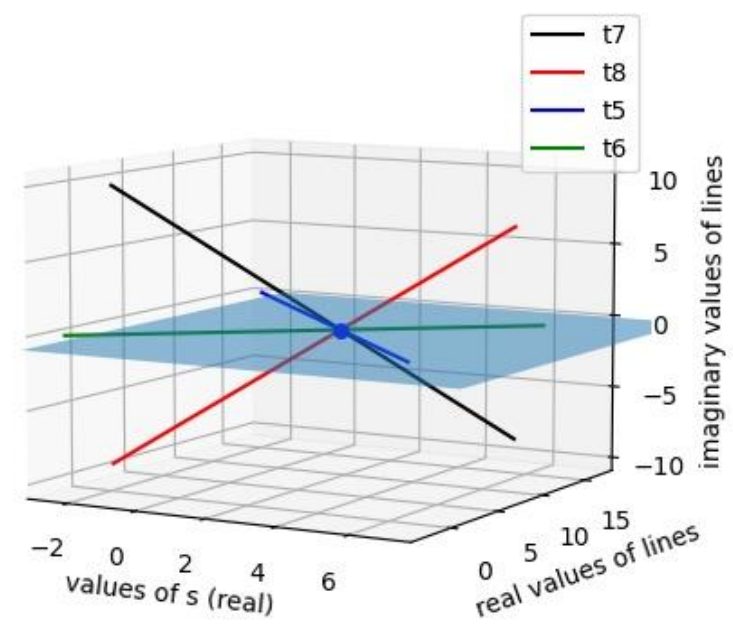

Fig.11.4.1 - All lines in this plotting obey the Meeting Point Law, crossing at the blue dot on the real plane $z=0$. The real plane is rendered in bluish gray. Real lines t5 (green) and t6 (blue) lie on the real plane 
Plot of complex and real lines, with the real $(z=0)$ plane in transparent grey

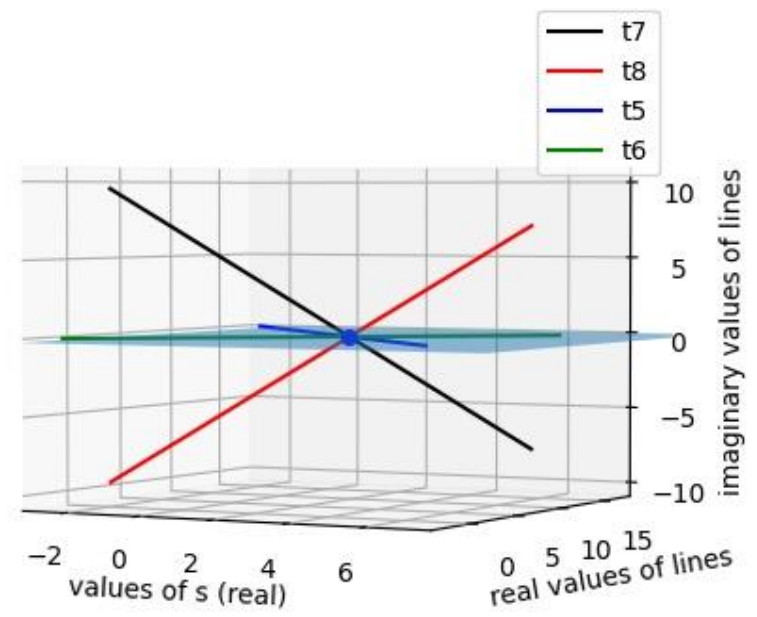

Fig.11.4.2 - The lines of preceding figure are shown from an observer's point of view positioned almost at the level of the real plane $z=0$. The complex lines stay in the complex space except when going thru the real plane at the blue dot

Plot of complex and real lines, with the real $(z=0)$ plane in transparent grey

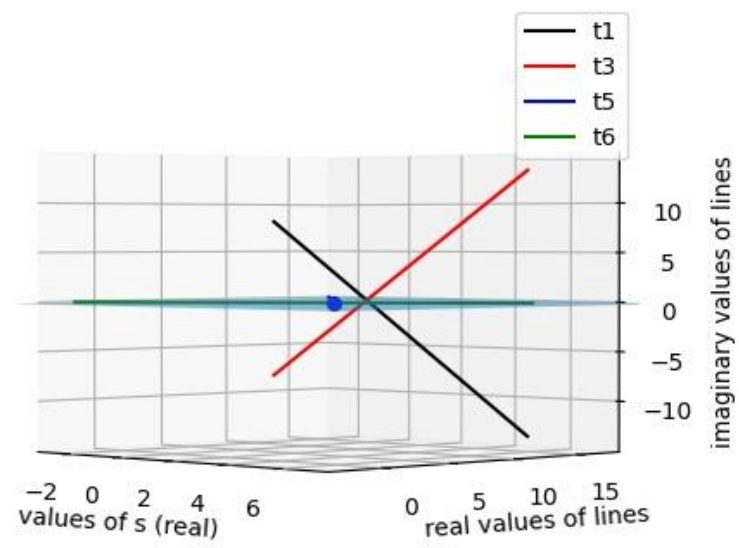

Fig.11.4.3 - With the same point of view as in the preceding figure, the pair t1 (black) and its conjugate $\mathrm{t} 3$ (red) cross the real plane but not on the blue dot, thus confirming that they do not obey the Law

Let us now see the contribution of method Nelder-Mead to the case of process YE.

Figure 11.4.4 shows a peculiar behavior. For positive initial values of $x$, the answer is $(x=1.63, y=1.42)$, as shown in Table 6.1 and also coinciding with solution 6 in Table 11.4. As soon as the initial value of $x$ becomes slightly negative, the answer jumps to 
( $x=-1.97, y=11.95)$, corresponding to solution 5. But the answer does not stay at this value forever: around an initial value of $x=-6.6$, the answer jumps back to the former value $(x=1.63, y=1.42)$ !

Variation of $(x, y)$ with initial value for minimization; $p=2$;

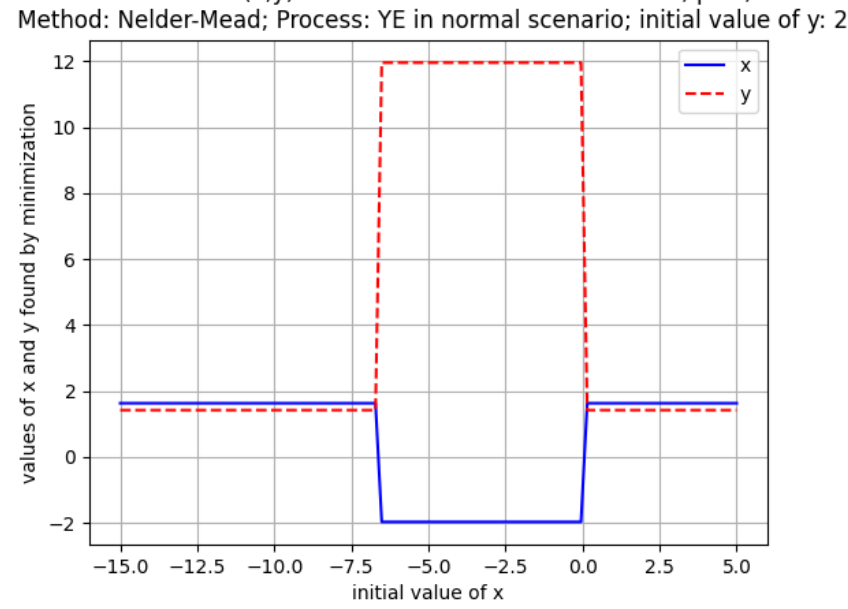

Fig. 11.4.4 - Process YE: depending on the initial value of $x$, minimization by NelderMead gives different answers for $x$ and $y$

\section{5 - Process PE}

Analytically, process PE resulted in two solutions, both of them obeying the Meeting Point Law:

$$
\text { solution 1: } t=1.45 s+1.96 \text {; solution 2: } t=-3.48 s+16.37
$$

The results from Nelder-Mead method are shown on Figure 11.5. For positive initial values of $x$, the answer is identical to solution 1 (also shown in Table 6.1). When the initial value of $x$ goes negative, in the beginning the answer stays at the same value, but when a certain critical point is reached, the answer jumps to a different solution, described as ( $x=$ negative values in order of magnitude of $-10^{16} ; y=$ positive values in order of magnitude of $10^{16}$ ). This means that the method failed to converge.

Zooming into the critical point, we found that it stays in the interval [-3.4642603 to -3.4622602]. It was not possible to obtain a value near solution 2, as it was expected.

For clarity, in the figure the values of magnitude $10^{16}$ have been limited to $x=-8$ and $y=7$. 
Variation of $(x, y)$ with initial value for minimization; $p=2$;

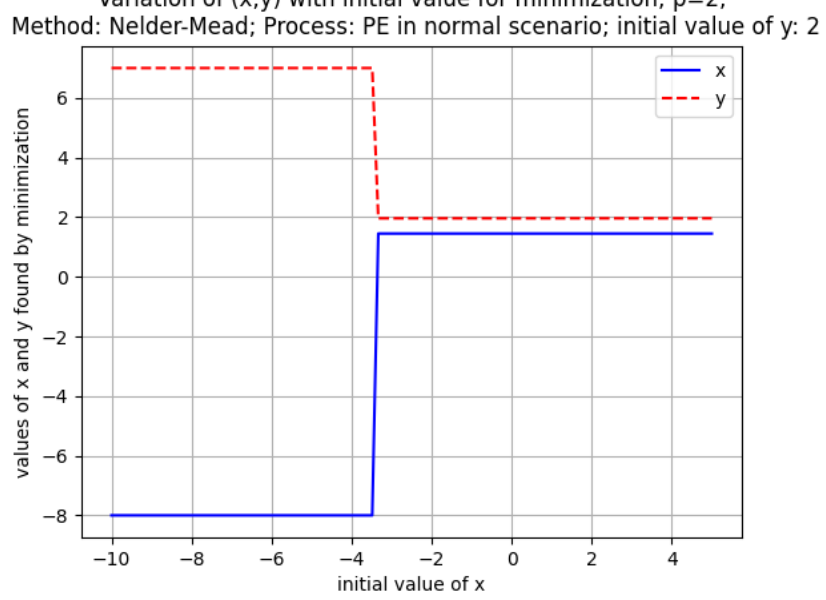

Fig. 11.5 - Process PE: depending on the initial value of $x$, minimization by NelderMead gives different answers for parameters $(x, y)$

\section{6 - Process AE}

Due to the restriction of keeping the absolute value of $x$ in the expression of process AE, it is not possible to analytically examine this process.

According to method Nelder-Mead, process AE repeats the peculiar behavior observed with process YE: when the initial values of $x$ are positive, the answer is solution 1 (the same as shown in Table 6.1); when the initial value of $x$ reaches zero, the answer jumps to solution 2. But when the initial value of $x$ goes deeper into negatives, suddenly the answers come back to solution 1. The behavior is shown in Figure 11.6, and the numerical values of solutions 1 and 2 are:

$$
\text { solution 1: } t=1.55 s+1.66 ; \quad \text { solution 2: } t=-1.55 s+10.73
$$

It should be noticed that both numerical solutions obey the Meeting Point Law!

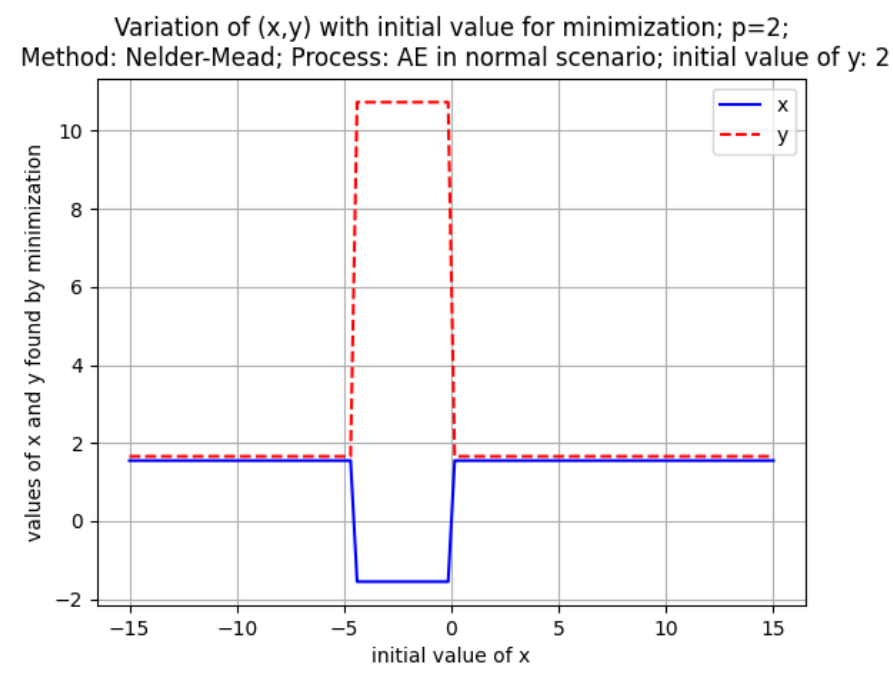

Fig. 11.6 - Process AE: depending on the initial value of $x$, minimization by NelderMead gives different answers for parameters $(x, y)$ 


\section{7 - Remarks}

We present some concluding remarks about what has been learned in this section 11 . The original idea was to check whether the processes with $p=2$ obey the Meeting Point Law (they all do), but in the way, we also got other precious information.

In the realm of $p=2$, the absolute value may be omitted and the minimizing expressions become continuous, differentiable functions, such that the minimization of errors may be analytically performed by elementary Calculus. The only exception is process $\mathrm{AE}$, where variable $x$ must be taken in its absolute value to avoid error cancellation.

We did the minimizations with the powerful Mathematica software, and we discovered that a few processes give multiple answers, some of these even being complex expressions.

Using the normal scenario, Mathematica also delivered numerical answers to the processes. Comparison of such results with those obtained by method Nelder-Mead are summarized in Table 11.7.

Nelder-Mead gives different answers depending on the starting values chosen for the line parameters. Such property makes somehow unreliable the use of these methods: can the answer be believed, or not? Is it a 'good' or a 'bad' answer?

It is worth observing that process PE gives a solution equal to the 'good' solution of Mathematica when initial $x$ stays in positive values. When entering negative values of initial $x$, however, Nelder-Mead switches to the 'bad' solution of Mathematica, then switches back to the 'good' solution again. This behavior suggests that the method does not invent multiple solutions by itself, only depending on the starting point. In fact, PE does have multiple solutions, as detected by Mathematica, and the method goes over all of them when the starting point is appropriately changed.

In practice, the problem is not too serious: if a certain answer gives a negative slope to the line, but the experimental points suggest a positive slope, then certainly this answer is 'bad'. Try a different initial point instead!

It was a fortunate case that, at the beginning of this study and yet without knowledge of all the intricacies of the processes, we had chosen initial values of minimizer such that 'good' values resulted for $x$ and $y$. Had we chosen otherwise, we could have prematurely concluded that the new proposed processes were useless, and this study would not exist! 
Table 11.7- Comparison of results of Mathematica (analytical) and Nelder-Mead (optimization, varying the initial value of $x$ ); initial value of $y: y=2$; normal scenario; $p=2, q=0$

\begin{tabular}{|c|c|c|c|}
\hline Process & Mathematica & $\begin{array}{c}\text { Nelder-Mead: } \\
\text { results from positive } \\
\text { values of initial } x\end{array}$ & $\begin{array}{c}\text { Nelder-Mead: } \\
\text { observed changes at cer- } \\
\text { tain values of initial } x\end{array}$ \\
\hline $\mathrm{VE}$ & $(x=1.29, y=2.41)$ & $(x=1.29, y=2.41)$ & none \\
\hline $\mathrm{HE}$ & $(x=1.86, y=0.75)$ & $(x=1.86, y=0.75)$ & $\begin{array}{c}\text { initial } x \approx 0 \rightarrow \\
\text { no convergence }\end{array}$ \\
\hline \multirow{2}{*}{$\mathrm{YE}$} & $\begin{array}{c}\text { solution 1: } \\
(x=1.63, y=1.42)\end{array}$ & $(x=1.63, y=1.42)$ & $\begin{array}{c}\text { initial } x \approx 0 \rightarrow \\
(x=-1.97, y=11.95)\end{array}$ \\
\cline { 2 - 5 } & $\begin{array}{c}\text { solution } 2: \\
(x=-1.97, y=11.95)\end{array}$ & & $\begin{array}{c}\text { initial } x \approx-6.6 \rightarrow \\
(x=1.63, y=1.42)\end{array}$ \\
\hline $\mathrm{PE}$ & $\begin{array}{c}\text { solution 1: } \\
(x=1.45, y=1.96)\end{array}$ & $(x=1.45, y=1.96)$ & $\begin{array}{c}\text { initial } x \approx-3.4: \\
\text { no convergence }\end{array}$ \\
\cline { 2 - 5 } & $(x=-3.48, y=16.37)$ & & $\begin{array}{c}\text { solution 2: } \\
\text { initial } x \approx 0 \rightarrow \\
\mathrm{AE}\end{array}$ \\
\cline { 2 - 5 } & & $(x=1.55, y=1.66)$ & $\begin{array}{c}\text { initial } x \approx-4.4 \rightarrow \\
(x=1.55, y=1.66)\end{array}$ \\
\hline
\end{tabular}

\section{The case of $p=1$}

\section{1 - Using method Nelder-Mead}

Choosing $p=1$ in the general expression means that errors are taken in their absolute value before summing. In this way, mutual cancellation of errors is avoided and the whole process represents a more natural way of finding the best line. But now the expressions contain a function (the absolute value) that is not differentiable everywhere, therefore preventing the use of elementary Calculus to minimize the total error in each process. It is not possible to use Mathematica to conduct an analytic minimizing process, so one has to relay entirely on methods like Nelder-Mead to do the job numerically.

The following results come from changing the initial value of $x$, as it was done for $p=2$ in the preceding section. As it is shown in Table 12.1 and Figures 12.1 to 12.5, everything goes in a fashion quite similar to the case $p=2$, as follows:

- Process VE gives the same line over a broad range $(-100,100)$ of initial values for $x$.

- Process HE does not converge for negative values of initial $x$.

- Process YE gives the 'good' line or the 'bad' line depending of initial $x$.

- Process PE shows perturbations even when initial $x$ is positive.

- Finally, process AE has a behavior similar to process YE, jumping from 'good' line to 'bad' line and back again to the 'good' line, as initial $x$ changes. 
Table 12.1-Results from varying initial value of $x ; p=1$;

Method: Nelder-Mead; normal scenario: $q=0 ; K=0.445$

(values of initial $x$ are approximate)

\begin{tabular}{|c|c|c|}
\hline Process & $\begin{array}{l}\text { Results from positive } \\
\text { values of initial } x\end{array}$ & $\begin{array}{l}\text { Observed changes at certain } \\
\text { values of initial } x\end{array}$ \\
\hline VE & $(x=0.95, y=4.05)$ & $\begin{array}{c}\text { no change observed in } \\
-100 \leq \text { initial } x \leq 100\end{array}$ \\
\hline $\mathrm{HE}$ & $(x=1.73, y=1.60)$ & $\begin{array}{c}\text { no convergence when } \\
\text { initial } x<0.051\end{array}$ \\
\hline \multirow[b]{2}{*}{ YE } & $(x=1.69, y=1.54)$ & $\begin{array}{c}\text { when }-0.62<\text { initial } x \leq 0 \\
(x=-1.94, y=9.88)\end{array}$ \\
\hline & & $\begin{array}{c}\text { itial } \mathrm{x}<-0.62, \text { it goes back to } \\
(x=1.69, y=1.54)\end{array}$ \\
\hline \multirow{3}{*}{$\mathrm{PE}$} & $\begin{array}{c}\text { mostly } \\
(x=1.33, y=3.33)\end{array}$ & $\begin{array}{c}\text { around initial } x=2.73 \\
(x=1.56, y=2.18), \text { then it goes } \\
\text { back to }(x=1.33, y=3.33)\end{array}$ \\
\hline & & $\begin{array}{c}\text { when }-8.6<\text { initial } x<3.8 \\
\text { no convergence }\end{array}$ \\
\hline & & $\begin{array}{l}\text { when initial } x<-8.6 \\
(x=-78, y=237)\end{array}$ \\
\hline \multirow[b]{2}{*}{$A E$} & $(x=1.69, y=1.54)$ & $\begin{array}{c}\text { when }-4.1<\text { initial } x<0, \\
x=-1.45 ; y=8.9\end{array}$ \\
\hline & & $\begin{array}{l}\text { when initial } x<-4.1 \\
\text { it goes back to } \\
(x=1.69, y=1.54)\end{array}$ \\
\hline
\end{tabular}

Variation of $(x, y)$ with initial value for minimization; $p=1$;

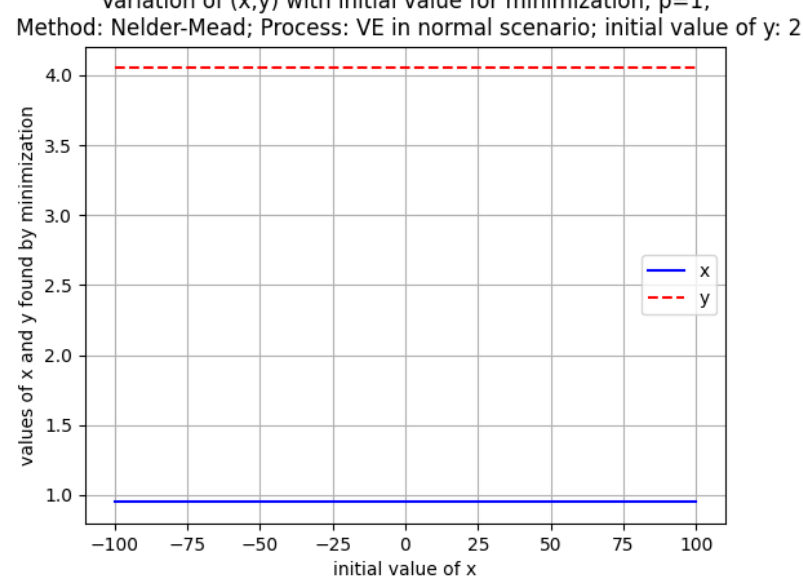

Fig. 12.1 - Process VE: stable solution of parameters $(x, y)$ when varying initial values of $x$ 
Variation of $(x, y)$ with initial value for minimization; $p=1$; Method: Nelder-Mead; Process: HE in normal scenario; initial value of $y: 2$

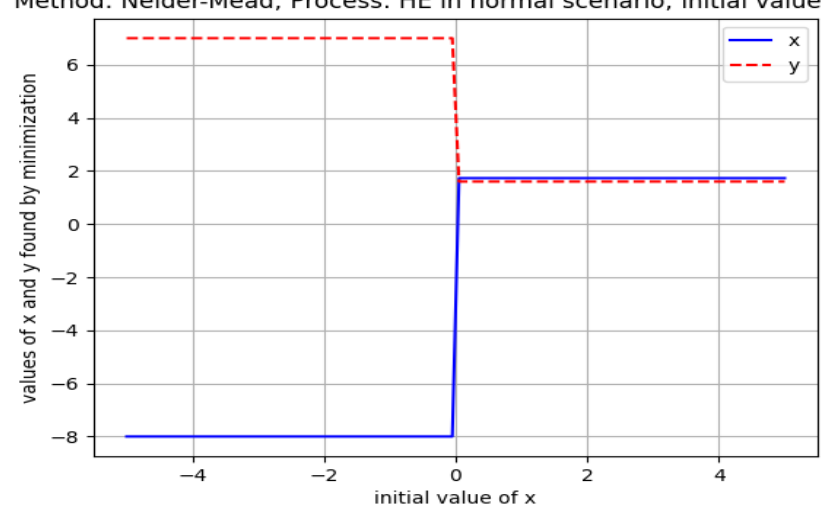

Fig. 12.2 - Process HE: change of values in $(x, y)$ in response to changes in the initial value of $x$, by a Nelder-Mead minimization

Variation of $(x, y)$ with initial value for minimization; $p=1$; Method: Nelder-Mead; Process: YE in normal scenario; initial value of y: 2

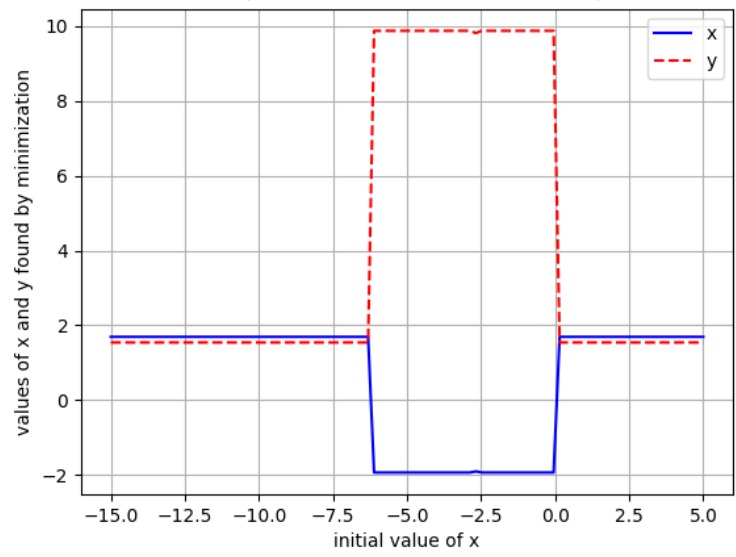

Fig. 12.3 - Process YE: depending on the initial value of $x$, minimization by NelderMead gives different answers for $x$ and $y$

Variation of $(x, y)$ with initial value for minimization; $p=1$; Method: Nelder-Mead; Process: PE in normal scenario; initial value of $y: 2$

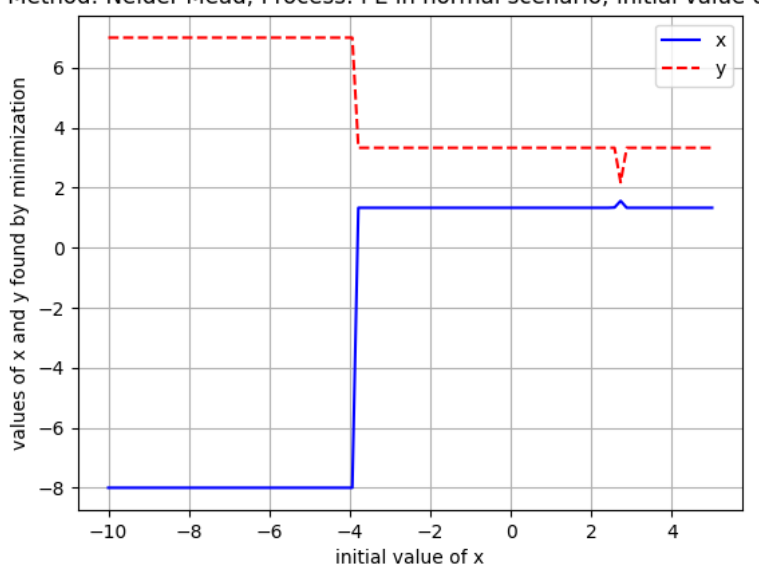

Fig. 12.4 - Process PE: depending on the initial value of $x$, minimization by NelderMead gives different answers for $x$ and $y$ 


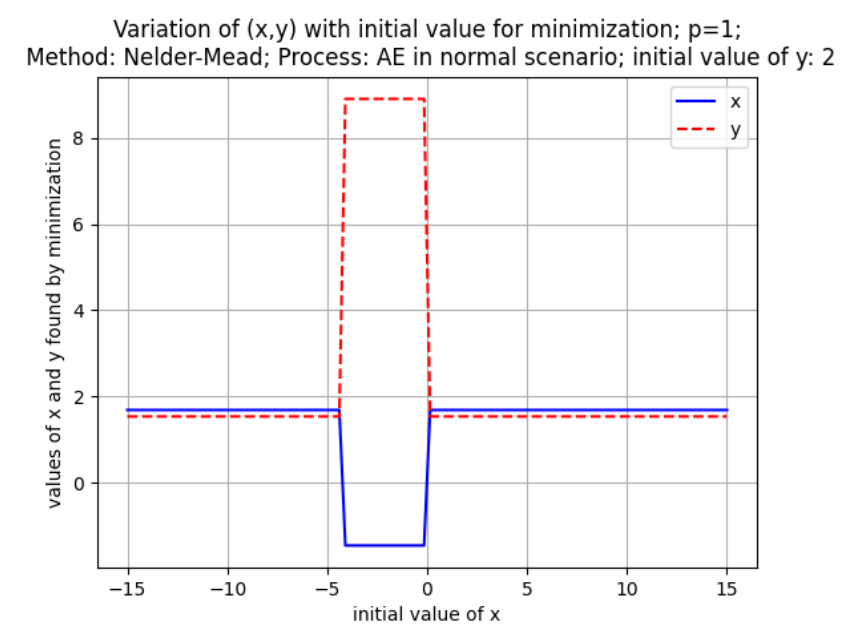

Fig. 12.5 - Process AE: depending on the initial value of $x$, minimization by NelderMead gives different answers for $x$ and $y$

\section{2 - The Meeting Point Law when $p=1$}

Is the Meeting Point Law a characteristic of $p=2$ only, or would it be encountered with $p=1$ also? Figure 12.6 shows a simple case: normal scenario, $p=1, q=0$ (results are shown in Table 12.2). There exist crossings of lines indeed, but not all at the same point. Therefore, the answer to the question is that with $p=1$ the Law does not hold; the Law is a property of $p=2$ only.

We should notice that the errors shown in Table 12.2 for the case $p=1$ (in units of $s$ ) cannot be directly compared with the error values for $p=2$ (in units of $s^{2}$ ) shown in Table 6.1.

Table 12.2 - Results for $p=1, q=0$; normal scenario; $K=0.445$

\begin{tabular}{|c|c|c|c|c|c|}
\hline Process & VE & HE & YE & PE & AE \\
\hline $\begin{array}{c}\text { Minimized } \\
\text { error value }\end{array}$ & 11 & 17 & 21 & 10 & 10 \\
\hline $\begin{array}{l}\text { Fitting line } \\
t=x . s+y\end{array}$ & $t=0.95 s+4.05$ & $t=1.73 s+1.60$ & $t=1.69 s+1.54$ & $t=1.33 s+3.33$ & $t=1.69 s+1.54$ \\
\hline
\end{tabular}




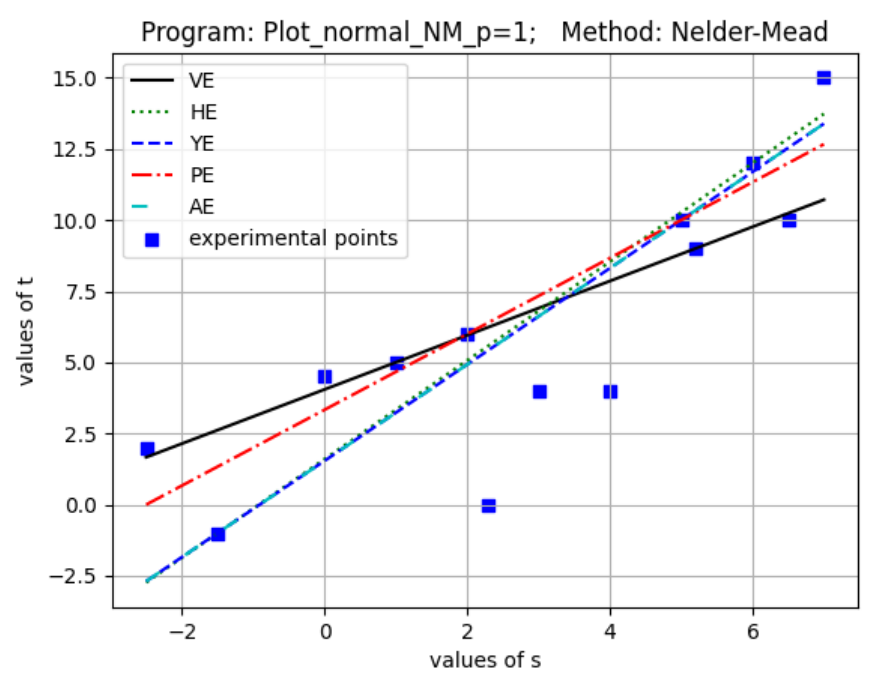

Fig. 12.6 - The Meeting Point Law does not hold when $p=1$

\section{The robustness against outliers}

The classic process and, generally, all processes with $p=2$ have been accused of displacing the best line when there are points far away from the nucleus of experimental points (the 'outliers'). The accusation has some fundament: since the errors are squared, the outliers attract the line towards themselves with greater force than other points do. That is, $p=2$ processes are not robust against outliers.

According with this reasoning, taking absolute values for avoiding mutual cancellation of errors instead of squaring would be a more natural way to determine the best fitting line and would assure robustness. In our study, it means taking $p=1$.

The reasoning is theoretically correct, but its praxis stumbles onto a difficulty: the absolute function is not differentiable everywhere, and most of minimizing methods do not like it. A popular method in the case is the Nelder-Mead algorithm, which we have used throughout this study.

The best way to evaluate robustness is to observe the lines both for $p=1$ and for $p=2$, on the same plot, with the same scenario with outliers studied in section 9, and for each process. Figures 13.1 to 13.5 dramatically show how $p=1$ processes are much more robust than $p=2$ processes. Table 13.1 contains the results obtained for $p=1$, for the scenario with outliers.

Table 13.1 - Results for $p=1, q=0$ with outliers; $K=0.155$

\begin{tabular}{|c|c|c|c|c|c|}
\hline Process & VE & HE & YE & PE & AE \\
\hline $\begin{array}{c}\text { Minimized } \\
\text { error value }\end{array}$ & 15 & 31 & 46 & 14 & 20 \\
\hline $\begin{array}{c}\text { Fitting line } \\
t=x . s+y\end{array}$ & $t=1.25 s+3.75$ & $t=23.53 s-54.12$ & $t=3.83 s-7.51$ & $t=1.33 s+3.33$ & $t=2.08 s+0.44$ \\
\hline
\end{tabular}




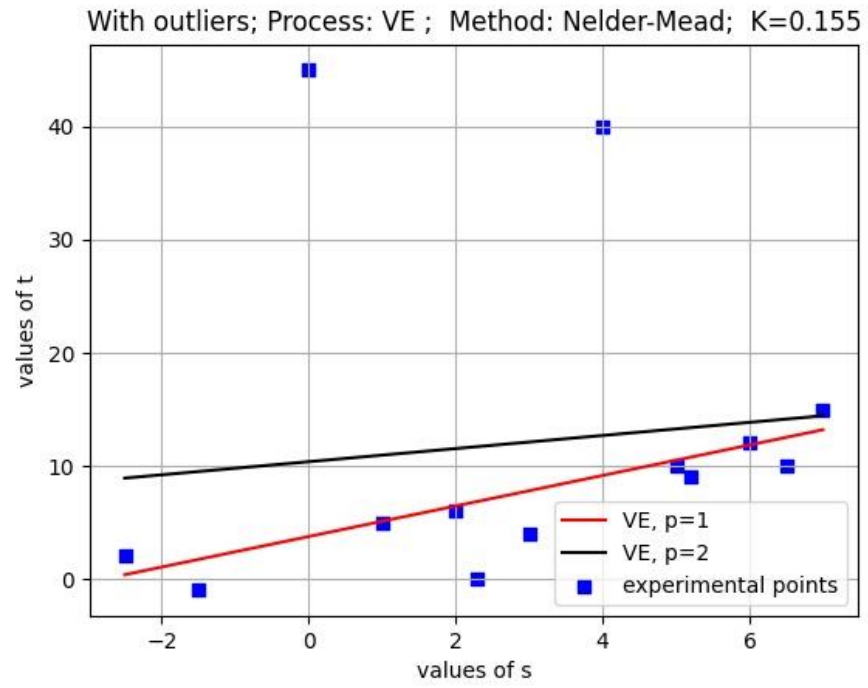

Fig. 13.1 - Process VE: comparison of fitting lines for $p=1$ and $p=2$. Experimental data with outliers

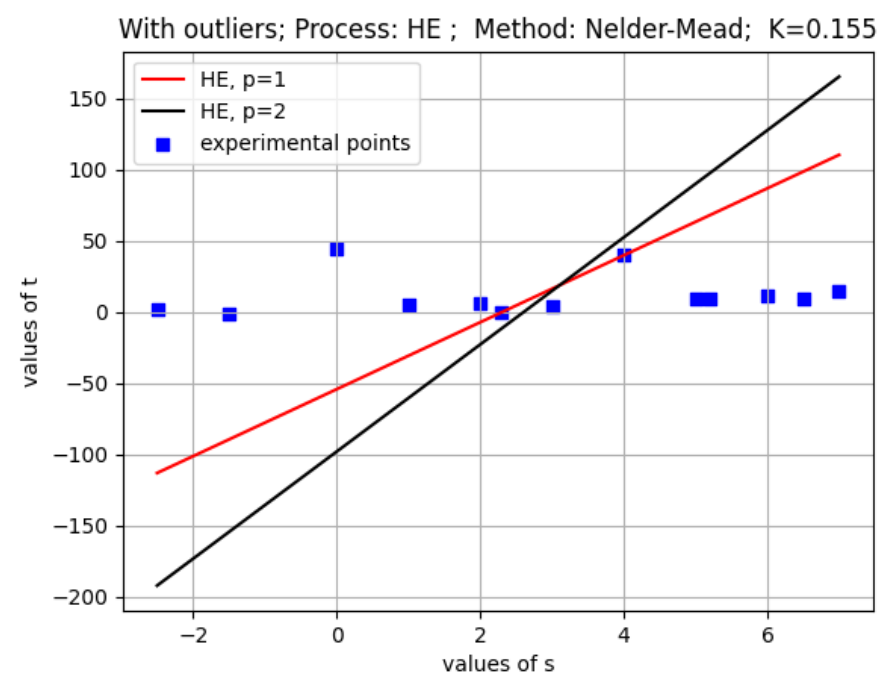

Fig. 13.2 - Process HE: comparison of fitting lines for $p=1$ and $p=2$. Experimental data with outliers 


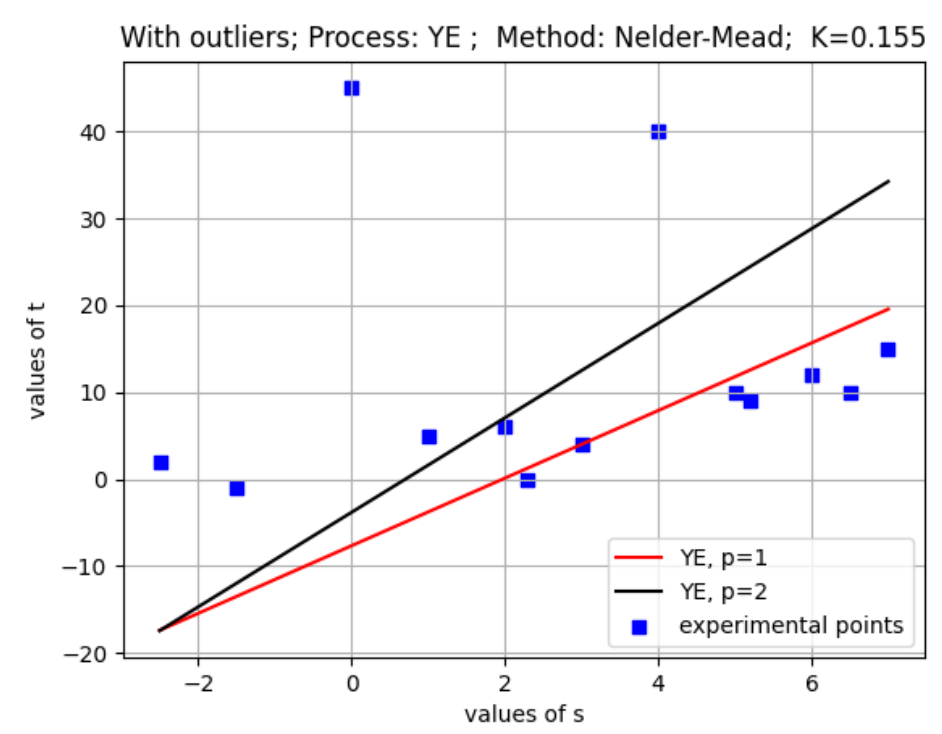

Fig. 13.3 - Process YE: comparison of fitting lines for $p=1$ and $p=2$. Experimental data with outliers

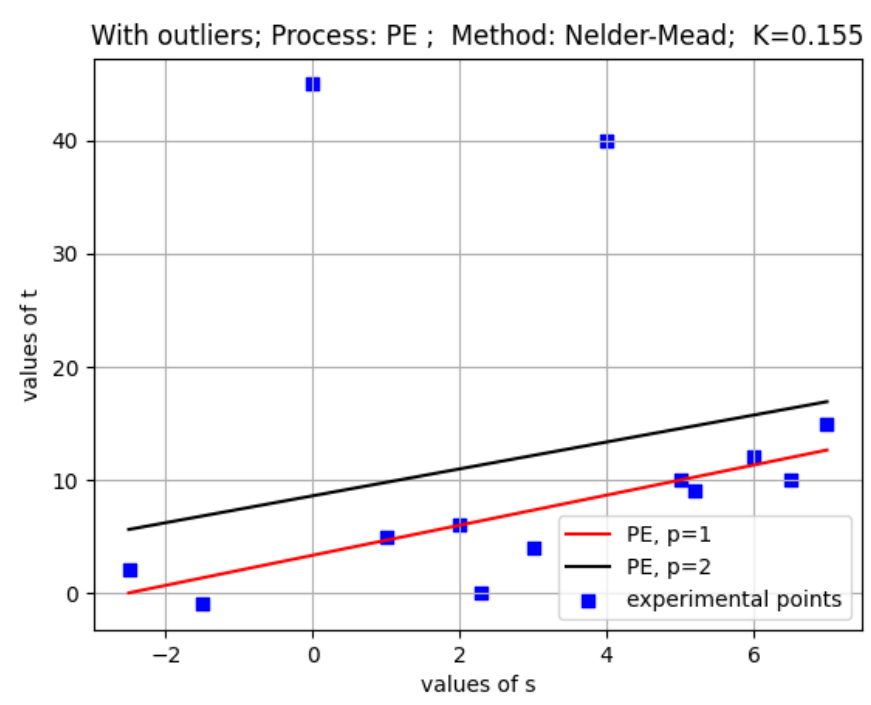

Fig. 13.4 - Process PE: comparison of fitting lines for $p=1$ and $p=2$. Experimental data with outliers 


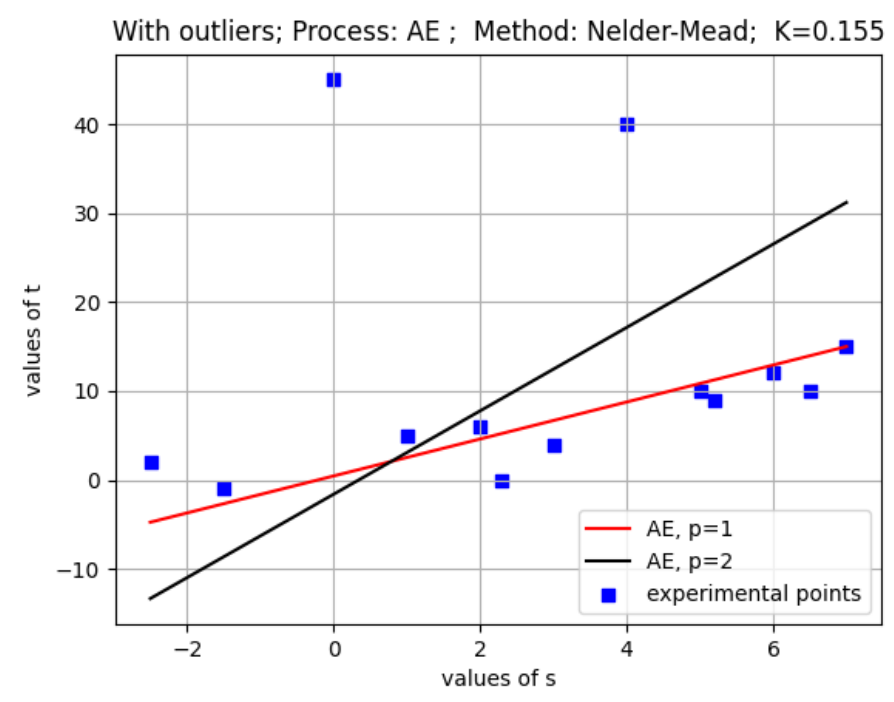

Fig. 13.5 - Process AE: comparison of fitting lines for $p=1$ and $p=2$.

Experimental data with outliers

\section{Concluding Remarks}

We end with a summary of what we learned from this extended study on the search for the 'best fitting line' through a set of experimental point.

We learned that it is possible and fruitful to define errors in ways different from the classical, centuries-old 'errors along the vertical line'. However, our general idea was similar to the old one: define individual 'error' as a function of each experimental point and a possible line (expressed as $t=x s+y$ ); obtain the 'best' line parameters (gradient $x$ and intercept $y$ ) by minimizing the total error (that is, the sum of all individual errors).

Considering the generic 'triangle of error' pictured in Figure 1.1, we defined five processes (five different criteria to evaluate the error), two of them taking errors along one coordinate axis only (VE, HE), and the remaining three processes taking errors from both coordinate axes (YE, PE, AE).

Specifically, the processes, as illustrated in Figure 4.1, can be described as follows: VE considers errors along the vertical axis (this is the 'classical' process); HE considers errors along the horizontal axis; YE uses the length of the hypotenuse as a measure of error; PE uses the height of the triangle as a measure of error; AE considers the square root of the triangle area as a measure of error.

For each process, an expression was written for the sum of the absolute value of individual errors elevated to an arbitrary exponent $p>0$.

Two facts called our attention. First, that the error functions for all processes could be written as the product of two other expressions, one (called 'factor $F^{\prime}$ ) exclusively dependent on the process itself and the exponent $p$, and the other one (called $S$ ) independent from the process but dependent on $p$ and on the experimental points. Computationally, this fact is useful, because if one wishes to compute all five processes corresponding to a given set of experimental points, then calculation of $S$ may be programmed in a common block for all processes. Besides, the presentation of the 
expressions becomes more elegant.

The second fact: in real life, values on coordinate axes usually have different physical dimensions, and so do the corresponding errors. This means that process VE delivers errors with the physical dimension of values on the vertical axis, and HE does it with the physical dimension of horizontal axis. How to numerically compare these two errors? And what about the processes that mix values from both axes, what is the dimension of their answers? In order to straighten up this question, and to assure that all computations are made with terms dimensionally compatible, a conversion factor $K$ is defined and computed. Our choice (arbitrary) in this study is that the dimension of results regards the horizontal axis. By using factor $K$, uniformity of physical dimensions is observed in all computations and numerical results from different processes can then be compared.

For the sake of generality, the expressions derived for the five processes allow arbitrary values of exponent $p$. However, only two values of $p$ have practical importance: $p=2$ and $p=1$.

The case $p=2$ corresponds to the 'classical' idea: individual errors are squared to avoid mutual cancellation; expressions are polynomials or rational functions, therefore differentiable at will; rules of elementary Calculus may be used to minimize the total error. The assumption of absolute values is unnecessary (except for process AE) and may be abandoned if using Calculus.

The case $p=1$ needs absolute values to avoid mutual cancellation of errors, but since the absolute function is not everywhere differentiable, such choice prevents the use of Calculus for minimization. Then minimization may be made only numerically by a method like the Nelder-Mead. The case $p=1$ is important when studying the robustness of the processes in the presence of outliers.

Our study begins with the case $p=2$. A basic set of thirteen experimental points was chosen; points' distribution suggests a best fitting line with positive gradient. The ordinates of the points may be changed, while leaving the abscissas without changes. The possibility of having outliers is also provided. Each arrangement of experimental points is called a scenario.

With this material, all processes have been tried with almost all scenarios, using method Nelder-Mead as minimizer.

Then, the minimized error was used as a comparison to choose the processes with better performance. On average, two processes that consider errors along both axes (PE, AE) resulted in the smallest minimized error and may be considered the best processes. Processes that rely on error along a single axis (VE, HE) stay at second place. Strangely, process YE gave the worst results; it seems that the length of hypotenuse is not a good way to evaluate errors.

All results are shown as Table-plus-Figure sets, one for each scenario. In the figures, it may be observed that all five best lines, corresponding to the five processes, intercept at the same point (for $p=2$ ). That is, for each scenario there is one point common to all best lines. Further investigation showed that such point is the 'center of mass' of the experimental point distribution. We then provisionally declared the existence of a 'Meeting Point Law', experimentally discovered. 'Provisionally', because we used specific scenarios, a specific minimizer. Who knows whether other scenarios or/and minimizers exist, not obeying such Law? In order to claim generality (for $p=2$, at least) of the Law, 
we decided to use the software Mathematica to minimize each process' expression analytically with the tools of Calculus (excepting process AE), then verify, always analytically, whether the Law is obeyed or not. If it would result that the Law is obeyed, then the Law may be considered as a universal Law (within $p=2$ ), not depending on scenario or minimizer.

Doing as planned brought some surprises, as revealing the existence of multiple solutions when minimizing certain processes, and even the possibility of complex solutions.

As a general result, we found that real solutions obey the 'Meeting Point Law'. Complex solutions always come in pairs (a solution and its complex conjugate) that cross, but not always obey the Law. Results from minimizers like Nelder-Mead may depend on the assumed initial values of $x$ and $y$. In practice, such incertitude does not impede the fruitful use of all processes: when two solutions are possible, if the solution at hand offers a negative $x$ when the shape of the experimental points' set suggests a positive $x$, then one can dump such 'bad' solution and change the initial values of $x$ and $y$ until the 'good' solution appears.

Finally, the case of $p=1$ was examined with Nelder-Mead. In this case, we verified that the processes do not obey the Meeting Point Law. Minimization of the error function was found to be also sensitive to the initial values of $x$ and $y$. Furthermore, graphical results confirm the greater robustness against outliers when $p=1$, as compared to $p=2$, for all processes.

\section{Acknowledgement}

The authors wish to thank Prof. Flavio A.M. Cipparrone for valuable suggestions to improve the manuscript. 